\title{
Energy Harvesting Irregular Repetition ALOHA With Replica Concatenation
}

\author{
Talha Akyıldiz ${ }^{\circledR}$, Student Member, IEEE, Umut Demirhan ${ }^{\circledR}$, Graduate Student Member, IEEE, \\ and Tolga M. Duman ${ }^{\circledR}$, Fellow, IEEE
}

\begin{abstract}
In this paper, we consider an asynchronous random access scheme called irregular repetition ALOHA (IRA) as a generalization of contention resolution ALOHA (CRA) with varying repetitions. We present an asymptotic performance analysis of CRA and IRA on the collision channel for regular and irregular repetition rates. We also propose an improvement by merging the clean parts of packet replicas in partial collisions, and extend our analysis to this scenario as well. Specific designs of repetition distributions based on the new analysis show that the optimized solutions of irregular repetition slotted ALOHA (IRSA) perform well in both IRA and the enhanced scheme, and they considerably outperform the regular repetition distributions. We also introduce energy harvesting (EH) to both schemes as a practical and sustainable adaptation, where users are able to harvest energy and store it in their finite-capacity batteries. We model the battery state by a discrete-time Markov chain and derive an optimal transmission policy to maximize the asymptotic performance of the system. We provide comprehensive numerical results for both practical and asymptotic scenarios to verify the validity of the proposed analyses, and illustrate the benefits of the proposed systems.
\end{abstract}

Index Terms-Random access, contention resolution ALOHA, irregular repetition ALOHA, asymptotic analysis, successive interference cancellation, energy harvesting.

\section{INTRODUCTION}

$\mathbf{W}$ ITH the foreseen proliferation of machine type devices (MTDs), massive machine-to-machine (M2M) communications have recently attracted great interest for future $5 \mathrm{G}$ systems. In massive M2M communications, the number of

Manuscript received February 18, 2020; revised July 29, 2020; accepted September 22, 2020. Date of publication October 14, 2020; date of current version February 11, 2021. The work of Talha Akyıldız was supported by the Scientific and Technological Research Council of Turkey (TÜBITAK) BIDEB 2210-A Scholarship Programme. The work of Tolga M. Duman was supported in part by the Scientific and Technological Research Council of Turkey (TÜBITAK) under Grant 119E589. This article was presented in part at the 2019 IEEE International Conference on Communications (ICC), Shanghai, China, May 2019. The associate editor coordinating the review of this article and approving it for publication was H. Pishro-Nik. (Corresponding author: Tolga M. Duman.)

Talha Akyıldız was with the Electrical and Electronics Engineering Department, Bilkent University, 06800 Ankara, Turkey. He is now with the Department of Electrical Engineering and Computer Science, University of Michigan, Ann Arbor, MI 48104 USA (e-mail: akyildiz@umich.edu).

Umut Demirhan is with the School of ECEE, Arizona State University, Tempe, AZ 85281 USA (e-mail: udemirhan@asu.edu).

Tolga M. Duman is with the Electrical and Electronics Engineering Department, Bilkent University, 06800 Ankara, Turkey (e-mail: duman@ee.bilkent.edu.tr).

Color versions of one or more of the figures in this article are available online at https://ieeexplore.ieee.org.

Digital Object Identifier 10.1109/TWC.2020.3029387 users is extremely high; users are only sporadically active in an unpredictable manner and they are equipped with low complexity and low energy consuming simple MTDs [2], [3]. These requirements pose compelling challenges that need to be overcome with a suitable access mechanism. Existing multiple access protocols, e.g., demand assignment multiple access (DAMA) and time division multiple access (TDMA), cannot meet the requirements of massive M2M communications due to signaling and control overhead which increases with network size. In addition, carrier sense multiple access (CSMA) demands steady channel sensing and some of its variants require a feedback mechanism to access the channel. Basic random access (RA) schemes, ALOHA and slotted ALOHA, allow users to share the channel without any coordination, however, they suffer from inevitable collisions that result in an inferior system performance. On the other hand, advanced random access (RA) approaches promise convenient and elegant solutions for M2M communications providing promising system performance, especially with the utilization of successive interference cancellation (SIC) techniques [4]. In this setting, while synchronous RA schemes provide better performance by taking the advantage of slot level synchronization, due to the challenges in achieving this, advanced asynchronous schemes are also appealing, and they should be investigated in detail.

In massive M2M communications, another important aspect is the long-term operation capability of the system as replacing batteries of massive number of MTDs is not viable, which brings about a need for energy-autonomous systems. To this end, energy harvesting (EH) appears as a promising solution, providing self-sustainability and perpetual operation with the energy harvested from the environment [5]. Moreover, thanks to the sporadic activity of the MTDs, a continuous use of high energy is not required, which allows MTDs to operate with low-energy consumption over the long-term making EH a favorable option for RA protocols. However, stochastic nature of available energy causes poor performance in standard RA schemes, and the design and analysis of such schemes should be carried out by paying attention to the energy limitations.

Advancements made to the predecessor RA schemes, namely ALOHA [6] and slotted ALOHA [7], via diversity [8] and SIC provide enormous improvement on the maximum throughput. For instance, contention resolution diversity slotted ALOHA (CRDSA) [4] is a slot-frame synchronous scheme where each user sends two packet replicas in each frame and 
the received packets are resolved through SIC among different slots, resulting in a maximum throughput of 0.55 packets per slot (as opposed to 0.36 for slotted ALOHA). Liva, in [9], proposes irregular repetition slotted ALOHA (IRSA), which generalizes CRDSA by varying the number of replicas, and optimizes the replica distributions via a graph-based asymptotic analysis to obtain (asymptotic) throughputs approaching 1. A more generic scheme, called coded slotted ALOHA (CSA) [10] replaces the repetition of packets with general linear block codes. ZigZag decoding is first proposed in [11] to solve the hidden terminal problem for wireless local networks, while it is also used for slotted ALOHA and carrier sensing multiple access [12], frameless ALOHA [13], and coded slotted ALOHA [14]. Many extensions on these advanced RA schemes for different scenarios are also studied in the literature (e.g., [15]-[22]).

Departing from the slotted ALOHA variants, to alleviate the strict synchronization requirements, the work in [23] proposes contention resolution ALOHA (CRA), which can be considered as an unslotted frame synchronous adaptation of CRDSA where each user sends a fixed number of replicas and the collisions are resolved through SIC. It also incorporates strong forward error correction (FEC) codes to resolve the colliding packets on the physical layer and demonstrates the resulting performance via simulations. Enhanced CRA (ECRA) is proposed in [24] extending the CRA by merging the parts of the packets with minimum interference (or no collision whenever possible) to utilize FEC codes more efficiently. The authors in [25] propose a fully asynchronous implementation of CRDSA, asynchronous contention resolution diversity ALOHA (ACRDA), resolving the collisions with FEC based on the signal-to-interference noise ratio (SINR) of the received packets (coupled with an approximate analysis). In [26], ECRA with different combining techniques is proposed, and an approximate performance bound for low channel loads coupled with simulation results is given. IRA with packet length diversity (IRA-PLD) is proposed in [27], which allows users to transmit their packets with varying durations as a source of further diversity in addition to the time diversity obtained via repetition of packets.

Regarding ALOHA based schemes adopting energy harvesting nodes, in [28], the authors study the effect of system parameters, i.e., contention window size, packet and energy arrival rates, in a slotted-ALOHA network with wireless energy harvesting nodes. They also consider the sum throughput maximization of the energy harvesting capable slotted-ALOHA with different transmission power policies in [29]. The stability region of two-node slotted ALOHA system with nodes having different energy harvesting rates and activation probabilities is investigated in [30]. In [31], the authors present the energy harvesting aware design and analysis of different MAC protocols, e.g., TDMA, framed-ALOHA (FA) and dynamic FA (DFA), in detail. Other related works on different MAC protocols with energy harvesting capable nodes can be found in [32], [33]. Another line of work [34] studies the approximate capacity of the energy harvesting channel with a finite battery. More recently, the authors in [35] propose energy harvesting IRSA (EH-IRSA) scheme by accommodating energy harvesting user nodes with a unit-sized battery, and then extend their work to the case of finite-sized battery equipped nodes in [36]. Specifically, they provide an asymptotic analysis of EH-IRSA by modifying density evolution according to the energy availability and design repetition distributions considering EH. We summarize the related works on ALOHA based RA schemes along with their main features in Table I. ${ }^{1}$

Although there are numerous works for asynchronous diversity ALOHA schemes, to the best of our knowledge, there is no study providing an asymptotic analysis for advanced asynchronous RA schemes in the literature. With this motivation, we present the design and analysis of asynchronous advanced RA protocols with various considerations. The main contributions can be summarized as follows:

- Development of an asymptotic analysis for CRA over the collision channel assuming that collisions are resolved through SIC. Initially, we consider regular repetition rates, and then we generalize our approach to irregular repetition distributions. We refer to the latter scheme as irregular repetition ALOHA (IRA). ${ }^{2}$ Via the proposed analysis, we observe that introducing irregular repetition rates offers higher asymptotic throughputs.

- Advancement of CRA/IRA with concatenation of the clean parts of the packet replicas in partial collisions, named CRA/IRA with two replica concatenation (CRARC/IRARC), to improve the system throughput. We extend our earlier analysis, which is amenable for further extensions, to this scheme as well.

- Adaptation of IRA and IRARC to the case with energy harvesting nodes equipped with finite-sized batteries. We specify the system design parameters and provide an asymptotic analysis for this scenario by taking the stochasticity of energy arrivals into account. We derive the optimal transmission policy based on available energy in the battery, to maximize the asymptotic throughput.

- Illustration of comprehensive numerical results for both asymptotic and practical scenarios, and verification of the accuracy of the developed asymptotic analytical results through finite length simulations.

The paper is organized as follows. In Section II, we describe the system model. Asymptotic analyses of CRA and IRA are developed in Section III. In Section IV, the analysis is extended to CRA/IRA with two replica concatenation. In Section V, the design and analysis of IRA and IRARC with energy harvesting are studied. Optimized repetition distributions and numerical results are given in Section VI. Finally, Section VII presents our conclusions.

\section{SySTEM MODEL}

\section{A. RA Scheme}

We consider a fully asynchronous random access scheme by removing stringent frame- and slot-level synchronization

\footnotetext{
${ }^{1}$ All listed advanced RA schemes utilize SIC and provide remarkable performance improvement over ALOHA, slotted ALOHA and diversity slotted ALOHA (DSA).

${ }^{2}$ IRA is a generalization of CRA with varying repetition rates. CRA can be considered as a special case of IRA with fixed repetition rates.
} 
TABLE I

Main Features of Related Aloha Based RA Schemes

\begin{tabular}{|c|c|c|c|c|}
\hline \hline RA scheme & $\begin{array}{c}\text { Frame } \\
\text { Asynchronous }\end{array}$ & $\begin{array}{c}\text { Slot } \\
\text { Asynchronous }\end{array}$ & $\begin{array}{c}\text { Irregular } \\
\text { Repetition Rates }\end{array}$ & Main Feature \\
\hline \hline CRDSA [4] & $x$ & $x$ & $x$ & Frame and slot synchronous scheme with regular repetition rates \\
\hline IRSA [9] & $x$ & $x$ & $\checkmark$ & Generalization of CRDSA with irregular repetition rates \\
\hline EH-IRSA [36] & $x$ & $x$ & $\checkmark$ & Adoptation of IRSA with energy harvesting nodes \\
\hline CRA [23] & $x$ & $\checkmark$ & $x$ & Slot asynchronous counterpart of CRDSA \\
\hline ACRDA [25] & $\checkmark$ & $\checkmark$ & $x$ & Fully asynchronous adaptation of CRDSA \\
\hline ECRA [26] & $\checkmark$ & $\checkmark$ & $x$ & Frame asynchronous enhanced version of CRA with combining techniques \\
\hline IRA & $\checkmark$ & $\checkmark$ & $\checkmark$ & Asynchronous RA scheme with irregular repetition rates \\
\hline IRARC & $\checkmark$ & $\checkmark$ & $\checkmark$ & IRA with replica concatenation \\
\hline EH-(IRA-IRARC) & $\checkmark$ & $\checkmark$ & $\checkmark$ & IRA and IRARC with energy harvesting nodes \\
\hline IRA-PLD [27] & $x$ & $\checkmark$ & $\checkmark$ & Extension of IRA with different packet lengths \\
\hline \hline
\end{tabular}

requirements among users. In our model, each user has its own local frame called virtual frame $(\mathrm{VF})^{3}$ and they are not synchronized. Each virtual frame has equal duration denoted by $T_{f}$ and it is the same for all the users. Users transmit a packet upon its arrival via its copies, called as replicas, within a VF. We assume that a user can have only one packet to be transmitted within a VF. ${ }^{4}$ All the packets and their copies have an equal duration denoted by $\tau$. The transmission time of the replicas are uniformly distributed over the duration of the VF. The packet replicas include the preamble sequences for the packet detection and channel estimation. Also, each packet replica contains the position information of other replicas inside the VF relative to its own location, e.g., with a pre-determined quantization of the time differences. We note that such quantization requires detection of the packets using common preamble sequences.

The beginning time of VFs is local to each user and determined with a random offset value after a packet is ready to be transmitted. We divide the time into consecutive VFs for each user. Nevertheless, the proposed model is still truly asynchronous since VFs are not synchronized among users. The users are sporadically activated with probability $\pi$ at the beginning of each VF. The activation probability is independent of users and VFs. Active users transmit their packets with a certain number of replicas, called as repetition rate, at different VFs. The repetition rate might be regular as in CRA with all active users transmitting a fixed number of replicas, or it can be drawn from a certain probability mass function (PMF) for each user in each VF as in IRSA. The total number of users, including both active and inactive users, is denoted by $N_{t}$. The number of active users, $N_{a}$, follows a binomial distribution, and the expected number of active users is denoted by $N$, i.e., $N=\mathbb{E}\left[N_{a}\right]=\pi N_{t}$. Even though the number of users is massive, because of the sporadic nature of the users, the active number of users at a certain time is limited, $\pi \ll 1$. We define the (normalized) channel load as the expected number of active users within the duration $\tau$, i.e.,

$$
G=\frac{\mathbb{E}\left[N_{a}\right] \cdot \tau}{T_{f}}=\frac{N \cdot \tau}{T_{f}}
$$

\footnotetext{
${ }^{3}$ The virtual frame concept is first presented in [25]. Neither in CRA [23] nor in the previous statement of IRA [1] VFs have been considered.

${ }^{4}$ Throughout the paper, we will refer to a user and a packet interchangeably.
}

We also define the load generated by multiple packet replicas, i.e., the physical load, denoted as $\mu$, as the expected number of packet replicas in an interval of length $\tau$, i.e., $\mu=d_{\mathrm{avg}} \cdot G$ where $d_{\mathrm{avg}}$ is the expected number of packet replicas per user. ${ }^{5}$

Finally, we define the packet loss rate (PLR), denoted by $P_{l}$, as the percentage of the packets (on average) that are not decoded at the receiver after all SIC iterations are completed, and the normalized throughput, $T$, as the expected number of successfully decoded packets in an interval of length $\tau$, i.e., $T=\left(1-P_{l}\right) G$.

\section{B. Channel Model and Decoding Process}

We adopt a multiple access collision channel, as typically considered in the analysis of RA schemes [9], where the receiver is able to detect whether a packet experiences collision or not. Collisions are destructive (unless the interference is removed), i.e., if a packet experiences interference, it cannot be resolved. Otherwise, it is always decoded successfully. We also assume that the receiver can distinguish the clean parts of the replicas for the replica concatenation scenario.

The receiver applies SIC for IRA over a sliding resolution window with size $W$ that spans multiple VFs. In each window, SIC iterations (also referred to as iterations) are applied as follows: 1) The receiver detects the packet replicas and estimates the channel information, in particular, the symbol timing, carrier frequency and phase, using the preamble and payload symbols. 2) The packets that are not in a collision are decoded. 3) The symbols of the decoded packets are regenerated by re-encoding and re-modulating the payload information and signaling bits. 4) With the help of channel and location information, the regenerated packets are used to remove the interference of the other replicas of that packet within the resolution window. ${ }^{6}$ The SIC iterations are applied until there is no collision-free replica in the current resolution

\footnotetext{
${ }^{5}$ We note that $G$ is defined by scaling the expected number of active users by $\tau / T_{f}$ without including replicas. $G$ and $\mu$ are defined for the duration of $\tau$ since our analysis is performed with respect to this quantity.

${ }^{6}$ We need to remark that as discussed in [4] and [25], the symbol timing and carrier frequency are identical for the replicas that belong to the same packet, however, this is not the case for carrier phase since it is uncorrelated among different replicas. The phase needs to be re-estimated for all the replicas which can be performed via correlation based techniques.
} 


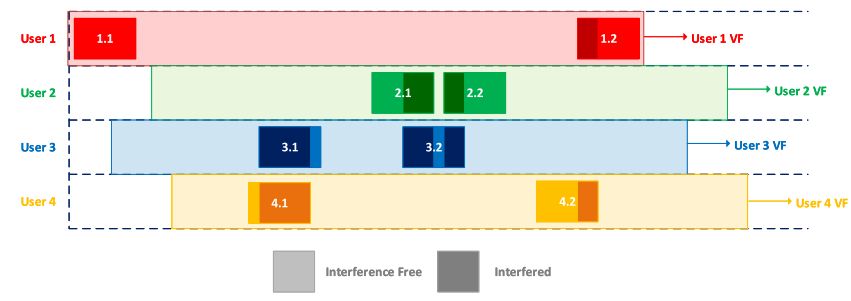

Fig. 1. An illustration of VF description and SIC procedure for IRA.

window or the maximum number of iterations is reached. After that, the resolution window is shifted with a duration of $\Delta W$ and the SIC mechanism is repeated again for the new window. Throughout the paper, we assume that channel parameters' estimation is perfect, and hence, interference cancellation is ideal. A more detailed discussion about imperfect (actual) SIC and parameter estimation can be found in [4] and [9].

For the enhanced scheme of IRARC, the receiver needs to concatenate the replicas with some clean portion to obtain a complete clean packet. We assume that the receiver can still detect a packet through the preamble sequence even under collision $^{7}$ and discriminate the clean parts of a packet using the in-phase and quadrature binary $( \pm 1)$ payload symbols. Next, the receiver can estimate the channel parameters with the preamble sequence and interference free payload symbols, and obtain a complete clean packet. Lastly, the SIC mechanism can take place with this clean packet. The proposed enhancement brings additional complexity and may increase decoding delay. However, the replica concatenation is only employed when there is no clean packet remaining in the current window (when the SIC process of IRA cannot progress any further). Hence, the replica concatenation is an additional process and its complexity increase is mostly limited. ${ }^{8}$

An exemplary scenario of an ideal SIC procedure for IRA is illustrated at Fig. 1 which depicts 4 active users transmitting 2 replicas within their corresponding VFs. First, it can be seen that the only interference free replica belongs to user 1 . The packets are decoded with the following SIC iterations: Iteration I) The receiver detects the interference-free replica and decodes the packet of user 1. Exploiting SIC, the interference of the second replica of user 1 is extracted from the received signal. Iteration II) User 4's second replica is now interference-free and can be decoded correctly. The replicas of user 4 are cleaned from the received signal. Iteration III) User 3's packet can be decoded successfully and its interference is removed. Iteration $I V$ ) User 2 is the only remaining user in the system and is also decoded successfully.

\section{Energy Harvesting System}

In the energy harvesting scenario, users are energy harvesting capable and equipped with finite-capacity batteries.

\footnotetext{
${ }^{7}$ As pointed out in [4] and [25], preamble collision is highly unlikely and with a small frequency or time off-set, the preambles can be identified surely. Alternatively, similar to ZigZag decoding, multiple preambles can be located on both ends of a packet.

${ }^{8}$ The additional complexity due to the replica concatenation is only to detect the clean symbols of the packets and the concatenation of these symbols which is not computationally demanding. We also limit the number of replicas to be used in concatenation to 2 to lower the complexity.
}

TABLE II

NOTATION TABLE

\begin{tabular}{|c|c|c|c|}
\hline \hline Notation & Description & Notation & Description \\
\hline \hline$T_{f}$ & Virtual frame duration & $T$ & Normalized throughput \\
\hline$\tau$ & Packet duration & $P_{l}$ & Packet loss rate \\
\hline$N$ & Expected number of active users & $\pi$ & Activation probability \\
\hline$G$ & Normalized channel load & $\alpha$ & Energy harvesting rate \\
\hline$\mu$ & Physical channel load & $B_{\max }$ & Battery capacity \\
\hline \hline
\end{tabular}

We denote the battery capacity by $B_{\max }$. In the proposed system model, we assume that only inactive users can store energy into their batteries during each VF. This model makes the analysis tractable as the energy arrivals and transmissions occur in different VFs and is well-justified for low energy harvesting rates and activity probabilities as the energy arrivals of the active users become very small. Users consume the energy stored (harvested during their inactivity) for transmission. Without loss of generality, we assume that each energy arrival brings one unit of energy, and a single replica transmission consumes one unit of energy as well. ${ }^{9}$ The stochastic nature of energy arrivals is governed by a Poisson process with rate $\alpha$ through each VF, i.e., $\alpha$ refers to the expected number of energy arrivals within a VF. ${ }^{10}$ Active users determine their transmission policy according to the battery state at the beginning of each VF in a probabilistic manner. To clarify, users draw the number of replicas to be transmitted within the VF from a certain PMF determined by the available amount of energy in the battery. For instance, users may prefer to deplete the harvested energy, or alternatively, conserve a certain amount for use in future VFs. We emphasize that the repetition rate is not directly drawn from a given PMF as in IRA/IRSA. Instead, it is now determined by the battery state and the transmission policy.

We present important notations in Table II.

\section{Asymptotic ANALYsis OF CRA/IRA}

In this section, we present an analysis for CRA and IRA. We consider the asymptotic setting $\left(N, T_{f} \rightarrow \infty\right)$ while keeping the channel load $G$, activation probability $\pi$ and packet duration $\tau$ constant. In this setting, the replicas become statistically independent from each other, and they have identical statistics (including the success and failure probabilities through the SIC iterations) allowing us to examine each replica separately, i.e., determining the behavior of a sample replica is sufficient to analyze the entire scheme.

\footnotetext{
${ }^{9}$ In general, energy consumption for a replica transmission is relatively high compared to the amount of harvested energy in short time periods. For example, a sensor node might consume mWs of power for transmission while receiving only uWs from RF harvesting. Our simplified EH model accounts for this imbalance of the energy consumption and arrival rates with a proper selection of EH rate $\alpha$.

${ }^{10}$ Even though the VFs are not synchronized globally, we still assume that each user has local VF timing for transmissions which enables us to divide the time into VFs and consider the battery state at these specific time points. This simplification is achievable with an external battery with low energy consumption and makes the problem tractable.
} 


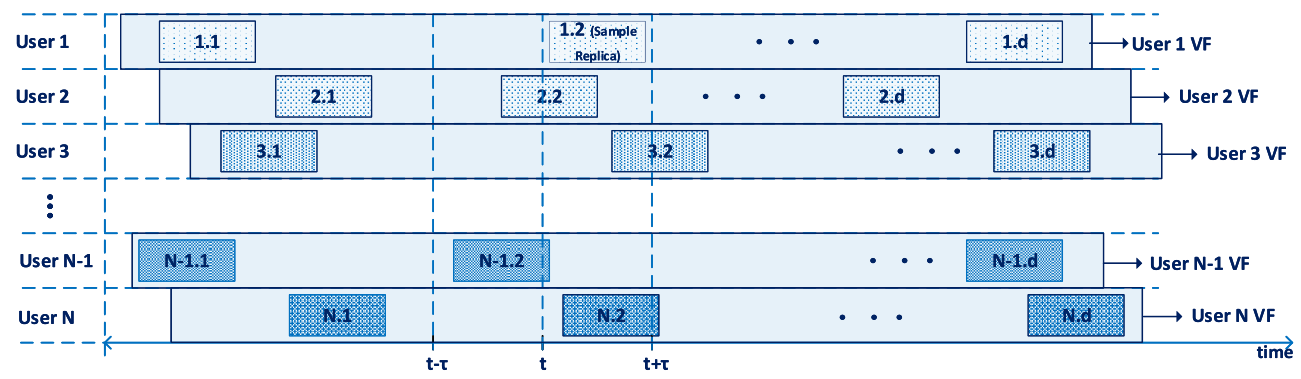

Fig. 2. Exemplary CRA VFs with $N$ users and $d$ replicas.

\section{A. Regular Repetition Rates (CRA)}

In this case, each user's packet is transmitted via $d$ replicas as illustrated in Fig. 2. Consider a specific packet and one of its replicas. We denote the probability of that replica not being resolved during iteration $i$ by $p_{i}$. We also define $q_{i}$ as the probability of the other replicas of that packet not being resolved at the previous iteration $(i-1)$. A replica is unknown only if none of its other $d-1$ replicas are resolved at the previous iteration, i.e., $q_{i}=p_{i-1}^{d-1}$. Similarly, a replica cannot be decoded during iteration $i$ if at least one of the $l-1$ interfering packets are not subtracted from the received signal, i.e., $p_{i}=1-\left(1-q_{i}\right)^{l-1}$. To clarify, we refer to Fig. 2. The packet replica 2.2 carries erasure at the current iteration if all of the remaining replicas are not decoded at the previous one. Similarly, the sample replica 1.2 is erasure free only if all the replica arrivals inside the vulnerable period are known. Using these relationships, we can write the probability of a replica not being resolved recursively as $p_{i}=1-\left(1-p_{i-1}^{d-1}\right)^{l-1}$. Throughout the analysis, we will refer to $\mu_{i}$ as the remaining (unresolved) load at iteration $i$, i.e., $\mu_{i}=\mu q_{i}$.

We now compute the probability of $l-1$ packet arrivals inside the vulnerable period of the sample replica, i.e., the replicas that arrive in a specific interval of length of $2 \tau$ colliding with the reference replica. A user transmits a replica inside this vulnerable period with a probability of $2 \tau d / T_{f}$. Hence, we can write the probability of $l-1$ interfering replica arrivals colliding with the sample replica denoted by $\rho_{l-1}$ as

$$
\rho_{l-1}=\left(\begin{array}{c}
N \\
l-1
\end{array}\right)\left(\frac{2 \tau d}{T_{f}}\right)^{(l-1)}\left(1-\frac{2 \tau d}{T_{f}}\right)^{N-(l-1)} .
$$

Using the asymptotic setting, $\left(N, T_{f} \rightarrow \infty\right), \rho_{l-1}$ becomes a Poisson random variable with parameter $2 d G(2 \mu)$, i.e.,

$$
\rho_{l-1}=e^{-2 d G} \frac{(2 d G)^{(l-1)}}{(l-1) !}=e^{-2 \mu} \frac{(2 \mu)^{(l-1)}}{(l-1) !} .
$$

At the first iteration, none of the replicas are resolved (prior to the start of SIC, $p_{0}=q_{0}=1$ ), hence, for successful decoding of a chosen replica, there should not be any replica arrival inside the interval of length $2 \tau$, i.e., $p_{1}=1-\rho_{0}=$ $1-e^{-2 \mu}$.

Consider a specific packet replica after the execution of the first iteration. For the replica to be resolved, there should not be any unresolved replicas inside its vulnerable period. Hence, the remaining load at the second iteration, which is the expected arrival rate of unresolved replicas with respect to the specific replica, can be denoted as $\mu_{2}=\mu q_{2}=\mu p_{1}^{d-1}$. The load is scaled with the probability of a packet not being resolved in the first iteration which is only possible if the remaining $(d-1)$ replicas are not resolved. Hence, by using the remaining load, we write $p_{2}=1-e^{-2 \mu_{2}}$.

After describing the SIC procedure for iterations 1 and 2, we now write $p_{i}$ for an arbitrary iteration $i$. Recall that we have a recursive relationship $p_{i}=1-\left(1-p_{i-1}^{d-1}\right)^{l-1}$, which allows us to calculate the non-resolvability probability at the $i$-th iteration in terms of the $(i-1)$-st one. Hence, one can obtain $p_{i}$ by averaging over the $l-1$ replica arrival probability, $\rho_{l-1}$, i.e.,

$$
p_{i}=\sum_{l=1}^{\infty} \rho_{l-1}\left(1-\left(1-p_{i-1}^{d-1}\right)^{(l-1)}\right)=1-e^{-2 \mu_{i}} .
$$

\section{B. Irregular Repetition Rates (IRA)}

We now consider the scenario where the number of replicas are drawn from an arbitrary PMF. We define $\Lambda(x)$ as the user degree distribution, i.e.,

$$
\Lambda(x) \triangleq \sum_{d=1}^{d_{\max }} \Lambda_{d} x^{d}
$$

where $\Lambda_{d}$ denotes the probability of a user transmitting $d$ replicas and $d_{\max }$ is the maximum number of replicas. Average packet repetition rate can be found as $\Lambda^{\prime}(1)=\sum_{d} d \Lambda_{d}$, and $\mu=\Lambda^{\prime}(1) G$.

In order to extend the analysis in the previous subsection to this case, we again examine the receiver operations on a specific packet replica. We first compute the probability of a randomly selected replica belonging to a user with a repetition rate of $d$ as

$$
\lambda_{d}=\frac{d \Lambda_{d}}{\sum_{d=1}^{d_{\max }} d \Lambda_{d}} .
$$

We also define the replica degree distribution in polynomial form, $\lambda(x)$, as

$$
\lambda(x) \triangleq \sum_{d=1}^{d_{\max }} \lambda_{d} x^{d-1} \text { where } \lambda(x)=\frac{\Lambda^{\prime}(x)}{\Lambda^{\prime}(1)} .
$$

We need to update the definition of $q_{i}$, i.e., $q_{i}=p_{i-1}^{d-1}$, since active users might have different replica numbers. Therefore, we average over the replica degree distribution to obtain $q_{i}$ as

$$
q_{i}=\sum_{d=1}^{d_{\max }} \lambda_{d} \cdot p_{i-1}^{d-1}=\lambda\left(p_{i-1}\right)
$$




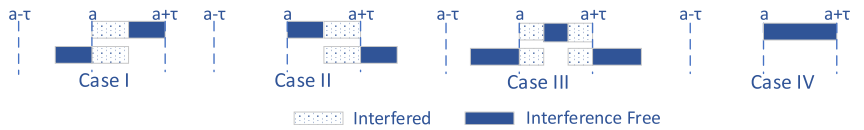

Fig. 3. Possible collision scenarios for a packet replica with some clean part.

The remaining load can also be expressed in terms of $G$ similar to CRA as $\mu_{i}=\Lambda^{\prime}(1) G \lambda\left(p_{i-1}\right)$, and we can rewrite $p_{i}$ in a generic form as follows $p_{i}=1-e^{-2 \mu \lambda\left(p_{i-1}\right)}=1-e^{-2 \mu_{i}}$.

Connection to PLR: Recalling the definitions of the packet loss rate and the normalized throughput, we write $P_{l}=$ $\Lambda\left(p_{i_{\max }}\right)^{11}$ and $T=\left(1-P_{l}\right) G$. We emphasize that these expressions are valid for both regular and irregular repetition rates. In the rest of the paper, we will continue only with IRA, as its analysis is more general, and covers that of CRA as well.

We also note that the provided analysis is amenable to different extensions although it is mainly developed for the destructive channel model. For instance, a packet can still be decoded with some level of interference relaxing the strict condition of collision channel with the use of FEC codes. To extend the analysis for such a scenario, the probability of decoding the colliding packets needs to be derived and incorporated into the analysis in a similar way to the works extending IRSA for different channel conditions and scenarios (e.g., [17], [20]). For instance, [23] adopts an SINR based decoding criterion which can be covered by our analysis by redefining the condition for successful recovery.

\section{IRA With Two RePlica CONCATENATION (IRARC)}

We now consider an extended version of IRA with SIC by concatenation of two overlapped replicas of a packet to build an interference free packet from the clean parts of colliding replicas. Our aim is to benefit from the partial collisions caused by asynchronism. We limit the number of replicas whose clean parts are combined to only two in order to keep the algorithm simple and examine the effect of this simplification in the numerical results section.

\section{A. Preliminaries}

There are four possible cases that a packet replica (with some clean portion) may encounter as illustrated in Fig. 3 . To explain, we divide the vulnerable period of the replica under consideration into two equal length intervals as $(a-\tau, a)$ and $(a, a+\tau)$ (where $a$ is the transmission start time of the packet). In case I, the packet is collided only from the left-hand side (LHS) meaning that there are colliding replicas starting in $(a-\tau, a)$ only. A similar argument holds for case II, i.e., the packet that is only collided from the right-hand side (RHS). In case III, the packet is collided from both sides at the same time. In addition to these three cases, completely interference free packets constitute case IV.

We now determine the amount of collisions from the LHS and RHS. We define $C_{k}^{-}$and $C_{l}^{+}$as the events of having $k$

\footnotetext{
${ }^{11}$ After all iterations completed, $p_{i_{\max }}$ is obtained representing the non-resolvability probability of a replica. For a packet (user) to be lost, all replicas should be unresolved, i.e., for a user with $d$ replicas, the probability of its packet not being decoded is $p_{i_{\max }}^{d}$. Since the user degree distribution is a polynomial representation of number of replicas, PLR can be written as $P_{l}=\Lambda\left(p_{i_{\max }}\right)$ averaging over the user degree distribution.
}

and $l$ colliding replica arrivals in the intervals $(a-\tau, a)$ and $(a, a+\tau)$, respectively. Therefore,

$$
\operatorname{Pr}\left\{C_{k}^{-}\right\}=\frac{e^{-\mu} \mu^{k}}{k !}, \quad \operatorname{Pr}\left\{C_{l}^{+}\right\}=\frac{e^{-\mu} \mu^{l}}{l !},
$$

as the arrivals follow a Poisson process with intensity $\mu$. For Poisson arrivals, conditioned on the number of arrivals in a given interval, the arrival time of the replicas are independent and identically distributed (i.i.d.) uniform random variables within their respective intervals. Hence, conditioning on the events $C_{k}^{-}$and $C_{l}^{+}$, we denote the arrival times of the replicas in $(a-\tau, a)$ and $(a, a+\tau)$ as $U_{1}^{-}, U_{2}^{-}, \ldots, U_{k}^{-}$and $U_{1}^{+}, U_{2}^{+}, \ldots, U_{l}^{+}$, respectively, and take $\tau=1$ for simplicity. We define the random variables $X, Y \in[0,1]$ as the maximum amount of collisions from the LHS and RHS, i.e.,

$$
X=\max _{1 \leq i \leq k} U_{i}^{-}, \quad Y=1-\min _{1 \leq j \leq l} U_{j}^{+} .
$$

The cumulative distribution function (CDF) and probability density function (PDF) of $X$ and $Y$ conditioned on $C_{k}^{-}$and $C_{l}^{+}$are: $F_{X}\left(x \mid C_{k}^{-}\right)=x^{k}, F_{Y}\left(y \mid C_{l}^{+}\right)=y^{l}, f_{X}\left(x \mid C_{k}^{-}\right)=$ $k x^{k-1}$, and $f_{Y}\left(y \mid C_{l}^{+}\right)=l y^{l-1}, x, y \in[0,1]$. Note that conditional PDFs and CDFs of $X$ and $Y$ are identical, and by using the total probability theorem, we can write

$$
F_{X}(x)=\sum_{k=0}^{\infty} \operatorname{Pr}\left\{C_{k}^{-}\right\} F_{X}\left(x \mid C_{k}^{-}\right)=e^{-\mu(1-x)}, \text { for } x \in[0,1] .
$$

Hence $f_{X}(x)=e^{-\mu} \delta(x)+\mu e^{-\mu(1-x)}(u(x)-u(x-1))$ where $u(x)$ is the unit step function, i.e., $X$ is a mixed random variable with a mass point at $x=0$. Note that for the asymptotic analysis, we utilize the continuous part of $X$. Hence, the conditional PDF and CDF defined as $f_{X \mid R}(x)=$ $\frac{\mu e^{-\mu(1-x)}}{1-e^{-\mu}}$ and $F_{X \mid R}(x)=\frac{e^{-\mu(1-x)}-e^{-\mu}}{1-e^{-\mu}}, x \in(0,1]$, with event $R=\{X \neq 0\}$ are also needed.

\section{B. Asymptotic Analysis}

We now provide an asymptotic analysis of IRA with two replica concatenation. We note that the success probability of case IV packets can be computed by the analysis of IRA presented in Section III. Hence, for the replica concatenation, we only need the additional resolvability probability (due to the packets that cannot be resolved by IRA alone). Hence, the objective is to find the successful concatenation probability of the unresolved packets using the clean portions of colliding replicas. Note that using only two partially colliding replicas for concatenation simplifies our analysis as case III replicas cannot be used to recover a packet in this scenario. ${ }^{12}$ Thus, these two replicas need to have collisions only from the LHS or RHS (cases I-II), respectively. Consider two replicas which belong to case I and II, respectively, as depicted in Fig. 4. Let $X_{1}$ and $Y_{2}$ denote the amount of collisions from the LHS and RHS of the two replicas, respectively. Free parts of these replicas are in the intervals $\left[X_{1}, 1\right]$ and $\left[0,1-Y_{2}\right]$. The successful recovery, i.e., $[0,1]=\left[X_{1}, 1\right] \cup\left[0,1-Y_{2}\right]$, is possible only if $X_{1} \leqslant 1-Y_{2}$.

\footnotetext{
${ }^{12}$ Utilization of case-III replicas require at least three replicas to be combined since they have collisions from both sides.
} 


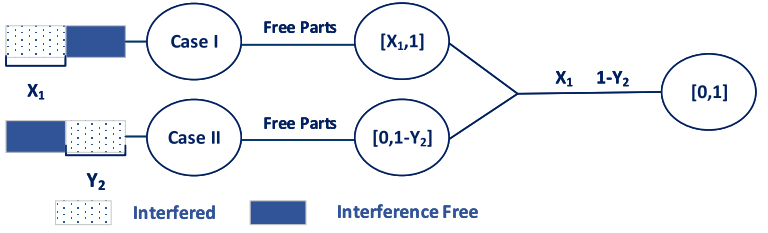

Fig. 4. A concatenation example with two replicas belonging to the cases I and II.

Let us consider a user with $d$ packet replicas. We denote the number of replicas experiencing case-I, case-II and case-III by $k, n$ and $d-n-k$ respectively. The selection of replicas can be made in $\left(\begin{array}{c}d \\ n, k\end{array}\right)=\frac{d !}{n ! k !(d-n-k) !}$ different ways. With $\xi(d, n, k)$ denoting the probability of $k, n$ and $d-n-k$ packets belonging to case-I, case-II and case-III, respectively. We write

$$
\begin{array}{r}
\xi(d, n, k) \\
=\left(\begin{array}{c}
d \\
n, k
\end{array}\right) \prod_{l=1}^{k} \underbrace{\operatorname{Pr}\left\{X_{l} \neq 0, Y_{l}=0\right\}}_{\text {case-I }} \prod_{m=k+1}^{n+k} \underbrace{\operatorname{Pr}\left\{X_{m}=0, Y_{m} \neq 0\right\}}_{\text {case-II }} \\
\cdot \prod_{s=n+k+1}^{d} \underbrace{\operatorname{Pr}\left\{X_{s} \neq 0, Y_{s} \neq 0\right\}}_{\text {case-III }} \\
=\left(\begin{array}{c}
d \\
n, k
\end{array}\right) \prod_{l=1}^{k} \operatorname{Pr}\left\{Y_{l}=0\right\} \operatorname{Pr}\left\{T_{k}\right\} \prod_{m=k+1}^{n+k} \operatorname{Pr}\left\{X_{m}=0\right\} \operatorname{Pr}\left\{S_{n}\right\} \\
\cdot \prod_{s=n+k+1}^{d} \operatorname{Pr}\left\{X_{s} \neq 0, Y_{s} \neq 0\right\}
\end{array}
$$

where $T_{k}=\bigcap_{l=1}^{k}\left\{X_{l} \neq 0\right\}$ and $S_{n}=\bigcap_{m=1}^{n}\left\{Y_{k+m} \neq 0\right\}$.

For successful concatenation probability, we first search for the replica with the largest clean part from the LHS that belongs to case-II, and then look for a second replica with the smallest colliding part from the LHS from case-I that we can concatenate with the first one. The amount of the smallest colliding part from the LHS among the $k$ replicas is denoted by $W_{k}$, i.e., $W_{k}=\min _{1 \leq l \leq k}\left(X_{l}\right)$ and the amount of largest clean part from the LHS among the $n$ replicas is denoted by $Z_{n}$, i.e., $Z_{n}=\max _{1 \leq m \leq n}\left(1-Y_{k+m}\right)$. Clearly, a successful recovery is possible, only if $W_{k} \leqslant Z_{n}$.

We define $\zeta(d, n, k)$ as the probability of successful concatenation for a user with $d$ repetitions given $k$ case-I, $n$ case-II and $d-n-k$ case-III packets. Then, $\zeta(d, n, k)$ can be written in terms of $\xi(d, n, k)$ by inserting the successful recovery condition $\left(W_{k} \leqslant Z_{n}\right)$ into (3):

$$
\zeta(d, n, k)=\xi(d, n, k) \cdot \operatorname{Pr}\left\{W_{k} \leqslant Z_{n} \mid T_{k}, S_{n}\right\} .
$$

As a next step, we need to calculate the probability $\operatorname{Pr}\left\{W_{k} \leqslant Z_{n} \mid T_{k}, S_{n}\right\}$. Note that the probability of $T_{k}$ and $S_{n}$ are $\operatorname{Pr}\left\{T_{k}\right\}=\left(1-e^{-\mu}\right)^{k}$ and $\operatorname{Pr}\left\{S_{n}\right\}=\left(1-e^{-\mu}\right)^{n}$. We determine the conditional CDF of $W_{k}$ and $Z_{n}$ conditioned on the events $T_{k}$ and $S_{n}$ as

$$
F_{W_{k} \mid T_{k}}(w)=1-\frac{\left(1-e^{-\mu(1-w)}\right)^{k}}{\left(1-e^{-\mu}\right)^{k}}, F_{Z_{n} \mid S_{n}}(z)=\frac{\left(1-e^{-\mu z}\right)^{n}}{\left(1-e^{-\mu}\right)^{n}},
$$

for $w, z \in(0,1]$. Using these, we obtain

$$
\begin{aligned}
& \operatorname{Pr}\left\{W_{k} \leqslant Z_{n} \mid T_{k}, S_{n}\right\} \\
& =1-\frac{n}{\left(1-e^{-\mu}\right)^{n+k}} \int_{0}^{1-e^{-\mu}}\left(1-\frac{e^{-\mu}}{1-u}\right)^{k} u^{n-1} d u,
\end{aligned}
$$

by simplifying the integral $\int_{0}^{1} F_{W_{k} \mid T_{k}}(z) f_{Z_{n} \mid S_{n}}(z) d z$. The integral in (5) can be written in terms of the Appell hypergeometric function $\left(F_{1}\left(a ; b_{1}, b_{2} ; c ; x, y\right)\right)[37],{ }^{13}$ i.e.,

$$
\begin{aligned}
& \int_{0}^{1-e^{-\mu}}\left(1-\frac{e^{-\mu}}{1-u}\right)^{k} u^{n-1} d u \\
& =\frac{\left(1-e^{-\mu}\right)^{(n+k)}}{n} F_{1}\left(n ; k,-k ; n+1 ; 1-e^{-\mu}, 1\right),
\end{aligned}
$$

hence,

$\operatorname{Pr}\left\{W_{k} \leqslant Z_{n} \mid T_{k}, S_{n}\right\}=1-F_{1}\left(n ; k,-k ; n+1 ; 1-e^{-\mu}, 1\right)$.

We can then rewrite $\zeta(d, n, k)$ by plugging (6) into (4) as

$$
\begin{aligned}
\zeta(d, n, k)= & \left(\begin{array}{c}
d \\
n, k
\end{array}\right) e^{-\mu(n+k)}\left(1-e^{-\mu}\right)^{(2 d-n-k)} \\
& \cdot\left(1-F_{1}\left(n ; k,-k ; n+1 ; 1-e^{-\mu}, 1\right)\right) .
\end{aligned}
$$

We also define $\zeta(d)$ as the total successful concatenation probability of a user with $d$ replicas in terms of $\zeta(d, n, k)$ by summing over all possible $n$ and $k$ values, i.e., $\zeta(d)=$ $\sum_{n=1}^{d-1} \sum_{k=1}^{d-n} \zeta(d, n, k)$.

To utilize these derivations in the analysis, we finally update the generic load $\mu$ with the remaining load at iteration $i$, $\mu_{i}$. For simplicity, we add a subscript $i$ to $\zeta($.$) , for any$ function $\zeta$ computed for the load $\mu_{i}$. We also need to define the probability of successful concatenation with respect to an unknown replica for iteration updates, denoted by $\bar{\zeta}_{i}(d)$, which can be written in terms of $\zeta_{i}(d)$ as $\bar{\zeta}_{i}(d)=\frac{\zeta_{i}(d)}{p_{i}}{ }^{14}$

We then specify $\zeta_{i}$ and $\bar{\zeta}_{i}$ as the expected success recovery probabilities for irregular repetition rates obtained by averaging $\zeta_{i}(d)$ and $\bar{\zeta}_{i}(d)$ over the user and replica degree probabilities, $\Lambda_{d}$ and $\lambda_{d}$, as

$$
\zeta_{i}=\sum_{d=1}^{d_{\max }} \Lambda_{d} \cdot \zeta_{i}(d), \quad \bar{\zeta}_{i}=\sum_{d=1}^{d_{\max }} \lambda_{d} \cdot \bar{\zeta}_{i}(d) .
$$

As the last step, we revise the remaining load defined in Section III as $\mu_{i}=\mu \lambda\left(p_{i-1}\right)$. Since, by performing replica concatenation, the receiver is now able to decode some additional packets among the remaining ones with probability $\bar{\zeta}_{i-1}$ at iteration $i-1$, the remaining load is updated as $\mu\left(\lambda\left(p_{i-1}\right)-\bar{\zeta}_{i-1}\right)$ for the $i$-th iteration. Finally, the PLR is updated as $P_{l}=\Lambda\left(p_{i_{\max }}\right)-\zeta_{i_{\max }}$ and $T=\left(1-P_{l}\right) G$ in a similar manner to IRA.

\footnotetext{
${ }^{13}$ The Appell hypergeometric function is defined in a generic form as, $F_{1}\left(a ; b_{1}, b_{2} ; c ; x, y\right)=\sum_{m=0}^{\infty} \sum_{n=0}^{\infty}\left(\frac{(a)_{m+n}\left(b_{1}\right)_{m}\left(b_{2}\right)_{n}}{m ! n !(c)_{m+n}}\right)\left(x^{m} y^{n}\right)$. The term $(x)_{n}$ is the Pochhammer symbol given by $(x)_{n}=\frac{\Gamma(x+n)}{\Gamma(x)}$.

${ }^{14}$ We note that $\zeta_{i}(d)$ is the total successful concatenation probability of a packet with $d$ replicas, while $\bar{\zeta}_{i}(d)$ is the conditional total successful concatenation probability on one of its unknown replicas. Therefore, we divide $\zeta_{i}(d)$ by $p_{i}$ (previously defined as failure probability of a replica).
} 


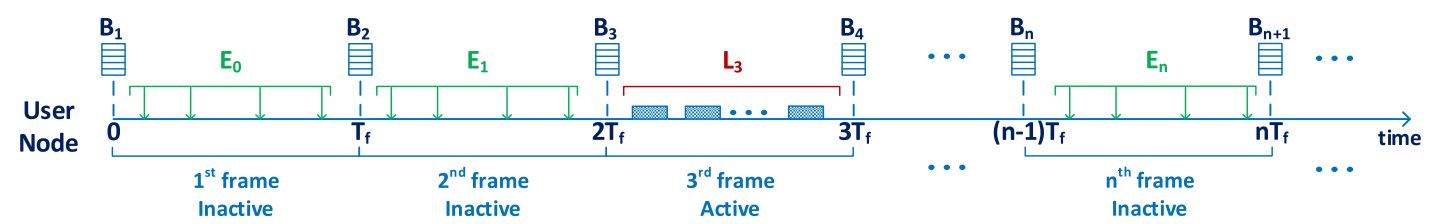

Fig. 5. A sample from consecutive virtual frames depicting both active and inactive state of a user node.

\section{ENERGY HARVESTING IRA AND IRARC}

We now consider a sustainable and practical adaptation of IRA and IRARC for energy harvesting nodes. Different from the previous schemes, energy for the transmissions is provided by the stored energy acquired via EH, therefore, the limitations and stochasticity of energy availability need to be taken into account. Specifically, the transmission of the replicas can only take place if there is stored energy in the battery. As a result, user nodes should satisfy the energy constraint, and the number of replicas should be chosen with respect to the available amount of energy at the beginning of a VF. We are interested in finding the optimal transmission policy for given battery state, that will be adopted by all of the active users in order to maximize the overall asymptotic system throughput.

\section{A. Asymptotic Analysis}

We present an asymptotic analysis for the EH scenario with a finite-sized battery. For the analysis, we first concentrate on determining the amount of energy available in the battery (battery state) for a given user node. We first argue that the steady-state distribution of the battery state can easily be computed for a given energy arrival rate $\alpha$, activation probability $\pi$, battery capacity $B_{\max }$ and transmission policy, defined as the transmission probabilities of replica numbers for each battery state. Next, we derive the repetition distribution based on the steady-state distribution of the battery state and the transmission policy.

A user node decides the number of replicas to be sent at the beginning of the VF. As the battery states at other time instants are not of interest, we define $B_{n}$ as the discrete-time stochastic process that represents the battery state at the beginning of the $n$-th VF. Recall that in our EH model, the harvested energy arrives stochastically, and follows a Poisson process with rate $\alpha$. We denote the number of energy arrivals in the $n$-th VF by $E_{n}$, and consequently, it is a Poisson random variable. We denote $e_{k}$ as the probability of $k$ energy arrivals in a VF, i.e., $e_{k}=\operatorname{Pr}\left\{E_{n}=k\right\}=\frac{e^{-\alpha} \alpha^{k}}{k !}, k=0,1,2, \ldots$.

At the beginning of each VF, a user selects the number of replicas to be transmitted probabilistically depending on the state of the battery, $B_{n}$. We note that the transmission policy is not specific to a VF, i.e., it is determined in advance and it is known by all the users. We denote the number of replicas that is sent within a VF by a random variable $L_{k}$ where $k$ units of energy is available in the battery, i.e., $L_{k} \leq k$ and $B_{n}=$ $k$. For ease of exposition we define $\gamma_{k}^{l}$ as the probability of transmitting $l$ replicas when the battery contains $k$ amounts of unit energy, i.e., $\gamma_{k}^{l}=\operatorname{Pr}\left\{L_{k}=l \mid B_{n}=k\right\}$, s.t. $\sum_{l=0}^{k} \gamma_{k}^{l}=1$, for $k \in\left\{0,1, \ldots, B_{\max }\right\}$.
Up to now, we have described the battery state, the energy arrival process and the transmission policy. The battery state at the next VF can be derived in terms of the current battery state, battery capacity (a constant), the number of unit energy arrivals (if the node is inactive) or the number of unit energy departures due to the transmissions (if the node is active). Hence, the battery state satisfies the Markov property, and it is possible to model the stochastic process $B_{n}$ as a discrete-time Markov chain. However, we have different processes for the active and inactive states of a user as depicted in Fig. 5.

During the inactive state of a user node, it only harvests energy, and does not attempt any transmissions within the VF. On the other hand, in an active state, a user node sends its packet replicas by utilizing the harvested energy in the battery. During the inactive state of a user, we can write $B_{n+1}$ in terms of $B_{n}$ and $E_{n}$ as $B_{n+1}=\min \left\{B_{n}+E_{n}, B_{\max }\right\}$ and the battery state evolves for an active state of a user with $k$ units of energy, $B_{n}=k$, as follows $B_{n+1}=B_{n}-L_{k}$.

Next, we define the one-step transition probabilities denoted by $\delta_{i j}$ as the probability that the battery is in state $j$ at $\mathrm{VF}$ $n+1$ given that the battery was in state $i$ at $\operatorname{VF} n$ for $i, j \in$ $\left\{0,1, \ldots, B_{\max }\right\}$, i.e., $\delta_{i j}=\operatorname{Pr}\left\{B_{n+1}=j \mid B_{n}=i\right\}$. This quantity can be written for the inactive state as

$$
\delta_{i j}= \begin{cases}\operatorname{Pr}\left\{E_{n}=(j-i)\right\}=e_{(j-i)}, & \text { if } j<B_{\max }, \\ \operatorname{Pr}\left\{E_{n} \geq(j-i)\right\}=\sum_{m=(j-i)}^{\infty} e_{m}, & \text { if } j=B_{\max },\end{cases}
$$

with the condition $j \geq i$ since a user node harvests energy only in the inactive state. Similarly, for the active state we can write the transition probabilities, i.e.,

$$
\delta_{i j}=\operatorname{Pr}\left\{L_{i}=(i-j)\right\}=\gamma_{i}^{(i-j)},
$$

with the condition $i \geq j$. Differently from the inactive state, the battery can have at most the same energy with the previous VF since the user node only transmits but does not harvest energy. Then, by utilizing (7) and (8) with the total probability theorem, we can write the overall one-step transition probabilities in a complete form as follows:

$$
\delta_{i j}= \begin{cases}(1-\pi) e_{(j-i)}, & \text { if } j>i \& j<B_{\max } \\ (1-\pi) \sum_{m=(j-i)}^{\infty} e_{m}, & \text { if } j>i \& j=B_{\max } \\ \pi \gamma_{i}^{(i-j)}, & \text { if } j<i \\ (1-\pi) e_{(j-i)}+\pi \gamma_{i}^{(i-j)}, & \text { if } j=i \& j<B_{\max } \\ (1-\pi) \sum_{m=(j-i)}^{\infty} e_{m}+\pi \gamma_{i}^{(i-j)}, & \text { if } j=i \& j=B_{\max } .\end{cases}
$$


Let $\mathbf{P}$ be the one-step transition matrix, where $i$-th row and $j$-th column element being $\delta_{i j}$, given as

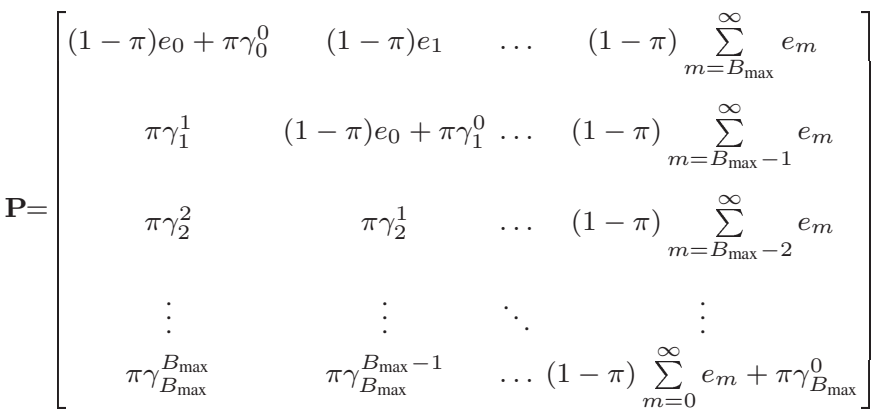

where $\mathbf{P} \in \mathbb{R}^{\left(B_{\max }+1\right) \times\left(B_{\max }+1\right)}$. As a next step, we can derive the steady-state distribution of the battery state denoted by $\boldsymbol{\beta}=\left[\beta_{0}, \beta_{1}, \ldots, \beta_{B_{\max }}\right]$, where $\beta_{i}=\lim _{n \rightarrow \infty} \operatorname{Pr}\left\{B_{n}=i\right\}$.

The steady state distribution can be calculated solving a system of linear equations, i.e., $\boldsymbol{\beta P}=\boldsymbol{\beta}$ with the following additional conditions, $\sum_{i=0}^{B_{\max }} \beta_{i}=1$ and $\beta_{i} \geq 0, \forall i$. We remark that steady state distribution, $\boldsymbol{\beta}$, depends on the energy arrival rate $\alpha$, the battery capacity $B_{\max }$ and the transmission policy probabilities, $\gamma_{k}^{l}$. As a last step, we note that we can compute the repetition distribution using the steady state distribution of the battery state and the transmission policy as $\Lambda_{d}=\sum_{k=0}^{B_{\max }} \beta_{k} \gamma_{k}^{d}$, for $d \in\{0,1, \ldots, k\}$, which enables us to perform the asymptotic analysis and evaluate the system performance via the steps already derived for IRA and IRARC in Sections III and IV, respectively.

We close this section by noting that asynchronous and coded RA schemes are not highly energy efficient in terms of the packet transmission attempts compared to synchronous and simple counterparts since redundant transmissions are made to achieve high throughputs, and the lack of synchronization trades the system performance with lower complexity (simple transmitters) and energy requirements for synchronizing massive number of users. Indeed, the main motivation to consider EH with RA is to address the stochasticity of energy arrivals, not to make the schemes more energy efficient.

\section{NumERICAL Results}

In this section, we present an extensive set of numerical examples. The section is divided into two parts. In the first part, we illustrate the asymptotic and finite-length simulation results of IRA and IRARC schemes with the optimized repetition distributions. In the second part, we study the performance of both schemes under the energy harvesting constraints with the optimized repetition distributions that take $\mathrm{EH}$ into account. We also discuss the effect of parameters $B_{\max }, \alpha$ and $\pi$ on the system performance and compare the performance of the optimal policy with the greedy and uniform policies as well.

\section{A. IRA and IRARC}

In this subsection, we utilize the developed asymptotic tools in Sections III and IV which enable us to determine the asymptotic $P_{l}$ and $T$ of IRA/IRARC for any given user
TABLE III

ASYMPTOTIC PERFORMANCE COMPARISON

\begin{tabular}{|c|c|c|c|}
\hline \hline User Degree Distributions $\Lambda(x)$ & $\begin{array}{c}\text { CRDSA- } \\
\text { IRSA (G*) }\end{array}$ & $\begin{array}{c}\text { CRA- } \\
\text { IRA (G*) }\end{array}$ & $\begin{array}{c}\text { CRARC- } \\
\text { IRARC (G*) }\end{array}$ \\
\hline \hline$\Lambda_{2}(x)=x^{2}$ & 0.541 & 0.271 & 0.344 \\
\hline$\Lambda_{4}(x)=0.5102 x^{2}+0.4898 x^{4}$ & 0.868 & 0.434 & 0.543 \\
\hline$\Lambda_{5}(x)=0.5631 x^{2}+0.0436 x^{3}+0.3933 x^{5}$ & 0.898 & 0.449 & 0.561 \\
\hline$\Lambda_{6}(x)=0.5465 x^{2}+0.1623 x^{3}+0.2912 x^{6}$ & 0.915 & 0.457 & 0.573 \\
\hline$\Lambda_{8}(x)=0.5 x^{2}+0.28 x^{3}+0.22 x^{8}$ & 0.938 & 0.469 & 0.59 \\
\hline \hline
\end{tabular}

degree distribution, $\Lambda(x)$, and search for the optimal distributions over possible load values. We aim at maximizing the asymptotic channel load, $G^{*}$, providing a vanishing $P_{l}$, for a given maximum repetition rate via differential evolution [38]. We define the maximum achievable asymptotic throughput $T^{*}$ as the corresponding throughput value for $G^{*}$, specifically, $T^{*}=G^{*}$ in this subsection since $P_{l}$ is very low. The optimized distributions for different maximum repetition rates and a regular distribution with repetition rate 2 along with their corresponding highest asymptotic channel load, $G^{*}$, for IRSA, IRA and IRARC are given in Table III. We observe that the ratio of maximum achievable throughput, $T^{*}$, between IRSA and IRA is $1 / 2$. We note that this asymptotic relationship is valid for both irregular and regular repetition rates, and it can also be observed through the analysis in Section III. Furthermore, our results show that the optimized distributions of IRSA perform well for both IRA and IRARC. ${ }^{15}$

The above behavior for the maximum achievable throughputs between IRSA and IRA deserves more explanation. The failure probabilities at each iteration for IRSA and IRA through the asymptotic analysis can be written as $p_{1, i}=$ $1-e^{-\mu_{1, i}}, p_{2, i}=1-e^{-2 \mu_{2, i}}$ with $\mu_{1, i}=\mu \lambda\left(p_{1, i-1}\right)$, $\mu_{2, i}=\mu \lambda\left(p_{2, i-1}\right)$ where $p_{1, i}$ is for IRSA and $p_{2, i}$ are for IRA. Due to the lack of slot synchronization, vulnerable period of a packet in IRA is doubled compared to IRSA. Note that for any given user degree distribution, $\Lambda(x)$ and $\lambda(x)$ are the same for the both cases. Thus, for $\mu_{1, i}=2 \mu_{2, i}$, their failure probabilities are equal, and we can write $G_{1, i}=2 G_{2, i}$ by using $\mu=\Lambda^{\prime}(1) G$. For the load values smaller than the decoding threshold $G^{*}$, the $P_{l}$ converges to a vanishing value. Then, by using $T^{*}=\left(1-P_{l}\right) G^{*}$ and $G_{1, i}^{*}=2 G_{2, i}^{*}$, one can obtain $T_{I R S A}^{*}=2 T_{I R A}^{*}$. Hence, it is not surprising that the throughput with IRA is half of that of IRSA. We note that while our analysis reveals this simple dependence between the throughputs of IRSA and IRA, this observation is not easy to make without the newly developed machinery.

A similar statement is not straightforward to make for IRARC. Intuitively, we only have a relatively small additional resolution probability due to concatenation that is derived through the same degree distributions, which may be the reason for the optimal distributions for IRA to execute well for IRARC as well.

\footnotetext{
${ }^{15}$ Differential evolution algorithm does not guarantee a globally optimal solution. Indeed, we found other distributions that perform slightly better for IRARC, however, we did not include them here as the improvements were very minor.
} 
We now perform finite length simulations for both IRA and IRARC. We evaluate various virtual frame lengths, i.e., 100, 200 and $1000 \mathrm{~ms}$, by assuming that all the replicas have the same length $\tau=1 \mathrm{~ms}$. We keep the maximum number of iterations $i_{\max }=20$, for all the simulations. We assume an ideal SIC throughout our simulations. The (finite) number of active users attempting transmission per each $\mathrm{VF}$ is $N=$ $G \cdot T_{f} / \tau$. The sliding resolution window size is chosen as $W=3 T_{f}$ and the decoding windows are shifted with $\Delta W=$ $0.15 T_{f}$.

We first provide the simulation results of IRA for $\Lambda_{8}(x)$ and CRA for $\Lambda_{2}(x)$ by using the above predefined virtual frame lengths in Fig. 6 along with the corresponding asymptotic analysis results. We also provide the performance of a basic ALOHA scheme to show the effectiveness of the SIC. The better fit of the finite length simulations and the asymptotic analysis is clearly seen for both throughput and $P_{l}$ as the virtual frame length increases for $\Lambda_{8}(x)$. While the analysis is carried out under the asymptotic setting, simulations are performed for finite length scenarios. In the asymptotic setting, each packet replica becomes statistically independent of each other, however, independence does not hold for finite frame lengths. The statistics of replicas become correlated with each other due to the occurrence of loops (stopping sets) which degrade the system performance, explaining the difference between the asymptotic and simulation results. We observe that the maximum achievable throughput, i.e., the decoding threshold is $G^{*}=T^{*}=0.469$. In addition, the irregular distribution $\Lambda_{8}(x)$ enhances the throughput by approximately $71 \%$ compared to the regular one $\Lambda_{2}(x)$, asymptotically. ${ }^{16}$ Notice that a similar enhancement is also observed for finite length simulations though the percentage improvement is smaller.

We now consider the same set-up with replica concatenation. Fig. 7 illustrates the finite length simulation results for $\Lambda_{8}(x)$ and $\Lambda_{2}(x)$ along with the asymptotic results of IRA with replica concatenation. We observe that the finite length simulation results comply with the asymptotic analysis, and that the decoding threshold $G^{*}$ is increased from 0.469 to 0.59 . That is, there is a significant improvement (by about $25 \%$ ) in throughput with replica concatenation. Furthermore, the use of irregular repetition rates offers superior performance as in CRA/IRA.

Fig. 8 depicts the simulation results of CRA and IRA with 2 and all possible replica concatenation with $T_{f}=$ $200 \mathrm{~ms}$. As noted earlier, introducing 2 replica concatenation results in a significant throughput increase compared to CRA/IRA, however, the simulation results show that concatenation of all the replicas performs only slightly better. We explain this as follows: for this specific example, $59 \%$ of the packets are resolved with only 1 replica (no concatenation), $30 \%$ with 2 replica concatenation, and $9 \%$ with 3 replica concatenation (while the remaining percentage

\footnotetext{
${ }^{16}$ For further comparisons, we also examine the asymptotic performance of regular and irregular distributions by keeping the maximum repetition rate fixed as 4 and 5. For the former, $\Lambda(x)=x^{4}$ and $\Lambda_{4}(x), G^{*}$ is increased from 0.386 to $0.434(\approx 12 \%)$. For the latter, $\Lambda(x)=x^{5}$ and $\Lambda_{5}(x), G^{*}$ is increased from 0.350 to $0.449(\approx 28 \%)$.
}
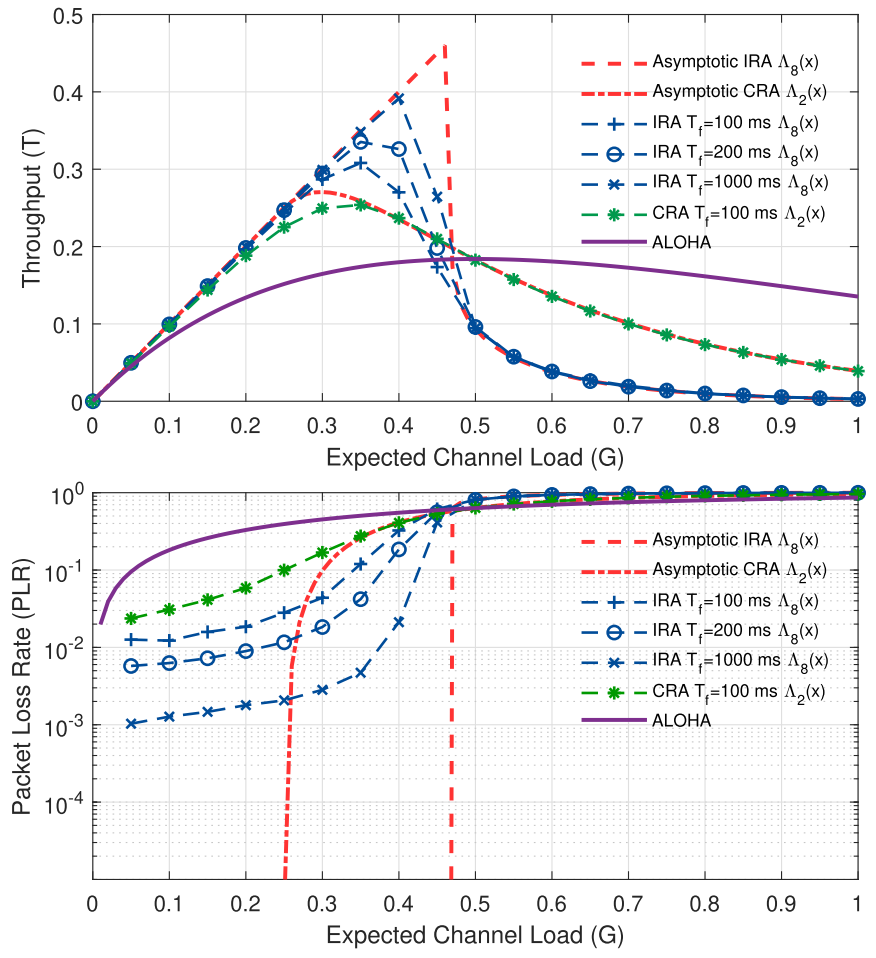

Fig. 6. Asymptotic analysis of CRA/IRA with $\Lambda_{8}(x)$ and $\Lambda_{2}(x)$ for different virtual frame lengths $\left(T_{f}=100,200,1000 \mathrm{~ms}\right), i_{\max }=20$.
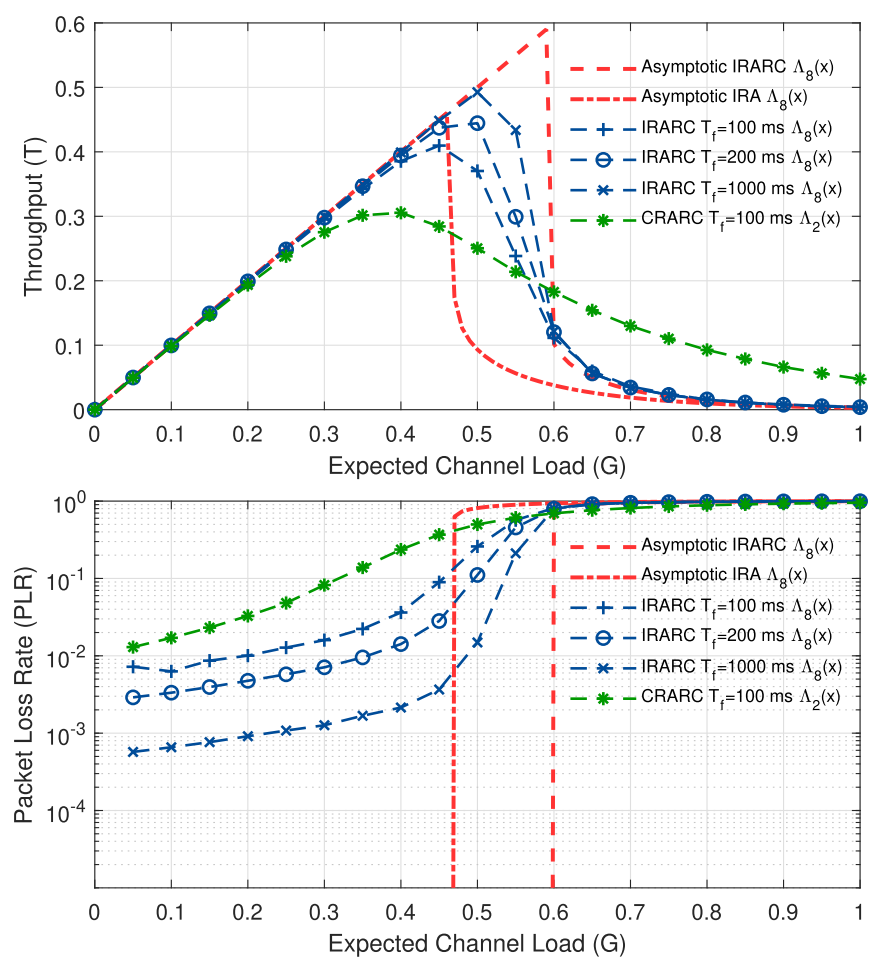

Fig. 7. Asymptotic analysis of IRARC and IRA with $\Lambda_{8}(x)$ for different virtual frame lengths $\left(T_{f}=100,200,1000 \mathrm{~ms}\right), i_{\max }=20$.

is due to concatenation of more replicas) at the specific load value of $G=0.5$. In other words, it may not be worthwhile to concatenate more than two replicas due to additional complexity, as most of the possible gain is already obtained with only two. Lastly, in the same plot, we also 


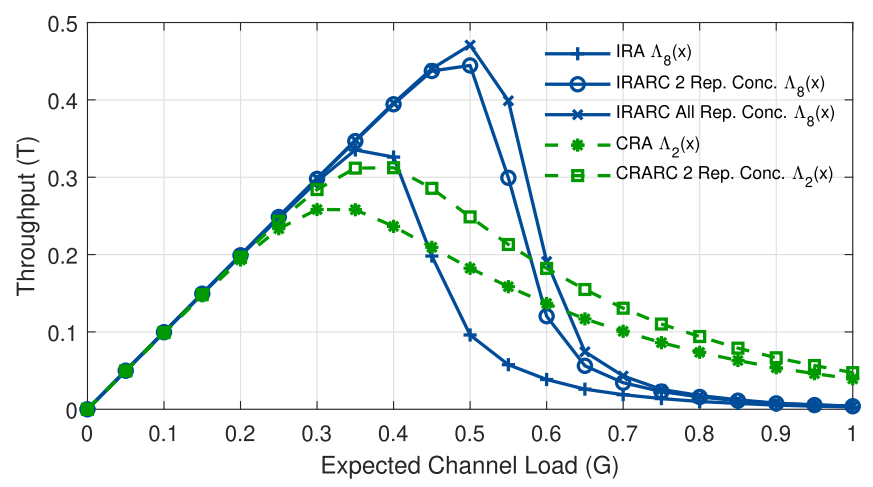

Fig. 8. CRA/IRA and CRARC/IRARC with 2 and all replica concatenation $\Lambda_{8}(x)$ and $\Lambda_{2}(x), T_{f}=200 \mathrm{~ms}, i_{\max }=20$.

include supplementary plots for CRA and CRARC with $\Lambda_{2}(x)$ to depict the additional resolvability, and hence, performance gain introduced by replica concatenation.

We also perform optimization with a constraint on the average repetition rate, $\Lambda^{\prime}(1)$. In other words, we limit the average transmission power of all active users for different values of $d_{\max }$ and perform differential evolution. The asymptotic performance $\left(G^{*}\right)$ of IRSA, IRA and IRARC for an average repetition rate of at most 3 , i.e., $\Lambda^{\prime}(1) \leq 3$, for different $d_{\text {max }}$ values is given in Table IV. We first note that for $d_{\max }$ values up to 5 the performance is identical to the unconstrained ones. On the other hand, for high values of $d_{\max }$, the performance of the schemes cannot improve any further due to the limit on the average repetition rate.

\section{B. EH-IRA and EH-IRARC}

We now illustrate the asymptotic and numerical results of the aforementioned unslotted RA schemes by accommodating energy harvesting nodes. For the EH RA schemes, we aim at determining the system parameters in order to maximize the asymptotic throughput, $T^{*}$, by employing the developed asymptotic tools in Sections III-V. Initially, we compute the resulting repetition distribution for given values of $B_{\max }$, $\alpha, \pi$ and most importantly the transmission probabilities, $\gamma_{k}^{l}$, by following the steps in Section V. Afterwards, the derived repetition distribution is used to determine asymptotic performance of the system using the developed asymptotic tools of IRA and IRARC in Sections III-IV. We note that the repetition distribution optimization is not straightforward as in IRA/IRARC, since the repetition distributions are determined by the steady-state of battery state and the transmission policy. Hence, to maximize the system performance, we optimize the transmission probabilities, $\gamma_{k}^{l}$, which results in optimized repetition distributions.

In this subsection, differently from the previous one, we perform optimization to maximize the asymptotic throughput, $T^{*}$, instead of maximizing $G^{*}$, without a constraint on $P_{l}$. The reasoning is that, in the case of $\mathrm{EH}$, packet losses are due to not only the collisions, as in IRA and IRARC, but also the packets that are not transmitted (at all) because of energy scarcity. Hence, obtaining vanishing $P_{l}$ values is not
TABLE IV

As ymptotic Performance of Distributions For MaXimum 3 AVERAGE REPETITION RATE

\begin{tabular}{|c|c|c|c|c|c|}
\hline \hline$d_{\max }$ & $\Lambda^{\prime}(1)$ & User Degree Distributions $\Lambda(x)$ & IRSA (G*) & IRA (G*) & IRARC (G*) \\
\hline \hline 3 & 2.81 & $\Lambda(x)=0.1882 x^{2}+0.8118 x^{3}$ & 0.824 & 0.412 & 0.513 \\
\hline 4 & 2.98 & $\Lambda(x)=0.5098 x^{2}+0.0026 x^{3}+0.4876 x^{4}$ & 0.868 & 0.434 & 0.543 \\
\hline 5 & 2.99 & $\Lambda(x)=0.5613 x^{2}+0.1619 x^{3}+0.2768 x^{5}$ & 0.887 & 0.443 & 0.56 \\
\hline 6 & 2.99 & $\Lambda(x)=0.5613 x^{2}+0.1619 x^{3}+0.2768 x^{5}$ & 0.887 & 0.443 & 0.56 \\
\hline 8 & 2.99 & $\Lambda(x)=0.5613 x^{2}+0.1619 x^{3}+0.2768 x^{5}$ & 0.887 & 0.443 & 0.56 \\
\hline \hline
\end{tabular}

TABLE V

Optimized Distributions for Given EnERgy ARrival RATE, $\alpha=$ $10^{-1}$, And ACTIVATION Probability, $\pi=10^{-2}$, For DifFEREnT BATTERY CAPACITY VALUES

\begin{tabular}{|c|c|c|c|}
\hline \hline$B_{\max }$ & User Degree Distributions $\Lambda_{B_{\max }}^{b}(x)$ & EH-IRA $\left(T^{*}\right)$ & EH-IRARC $\left(T^{*}\right)$ \\
\hline \hline 2 & $\Lambda_{2}^{b}(x)=0.1 x^{0}+0.08 x^{1}+0.82 x^{2}$ & 0.255 & 0.312 \\
\hline 3 & $\Lambda_{3}^{b}(x)=0.084 x^{0}+0.084 x^{1}+0.207 x^{2}+0.625 x^{3}$ & 0.403 & 0.512 \\
\hline 4 & $\Lambda_{4}^{b}(x)=0.027 x^{0}+0.092 x^{1}+0.081 x^{2}+0.79 x^{3}+0.01 x^{4}$ & 0.416 & 0.521 \\
\hline 5 & $\Lambda_{5}^{b}(x)=0.026 x^{0}+0.049 x^{1}+0.293 x^{2}+0.319 x^{3}+0.196 x^{4}+0.117 x^{5}$ & 0.434 & 0.541 \\
\hline 6 & $\Lambda_{6}^{b}(x)=0.023 x^{0}+0.035 x^{1}+0.339 x^{2}+0.38 x^{3}+0.086 x^{5}+0.137 x^{6}$ & 0.444 & 0.555 \\
\hline 8 & $\Lambda_{8}^{b}(x)=0.021 x^{0}+0.53 x^{2}+0.189 x^{3}+0.013 x^{4}+0.202 x^{6}+0.023 x^{7}+0.023 x^{8}$ & 0.459 & 0.578 \\
\hline \hline
\end{tabular}

guaranteed for $G \leq G^{*}$, therefore, $T^{*}$ is not necessarily equivalent to $G^{*}$. For instance, for low values of energy arrival rate, it is not possible to obtain low $P_{l}$ values since observing energy outages is more likely. In the optimization, we limit the search space with the load values up to the maximum asymptotic load obtained for the non-EH scenario, denoted as $\bar{G}$, and seek for optimal repetition distributions that result in the maximum throughput. Specifically, $\bar{G}$ depends on the battery capacity $B_{\max }$, and is selected as $G^{*}$ value in the corresponding non-EH schemes, provided by $\Lambda_{B_{\max }}(x)$. This approach helps with finding distributions with low $P_{l}$ values by eliminating the distributions with similar $T^{*}$ values for higher channel loads.

Some optimized distributions for different battery capacities, denoted as $\Lambda_{B_{\max }}^{b}(x)$, and given values of $\alpha=10^{-1}$ and $\pi=$ $10^{-2}$ with the corresponding asymptotic throughput values $T^{*}$ are listed in Table V. ${ }^{17}$ It can be seen that increasing the battery capacity enables the user nodes to store more energy, and hence, allows them to transmit more replicas improving the system performance in a similar way of increasing maximum repetition rate in IRA and IRARC. However, having higher battery capacity does not necessarily improve the throughput, since for low energy arrival rates, the full capacity may not be even utilized.

Next, we perform finite-length simulations to confirm the validity of the proposed asymptotic analysis. We adopt the same simulation set-up with the previous subsection. As an example, we depict the asymptotic and finite-length performance of the optimized distribution $\Lambda_{8}^{b}(x)$ with EH-IRA in Fig. 9. As can be observed, the simulation results conform with the asymptotic results obtained through the proposed analysis. It should be noted that for $G \leq G^{*}, P_{l}$ is a constant and equal to 0.02 , which is caused only by the user nodes experiencing energy absence, and in fact, the receiver is able to

\footnotetext{
${ }^{17}$ In the optimized distributions, $x^{0}$ term stands for active users not transmitting due to energy absence.
} 

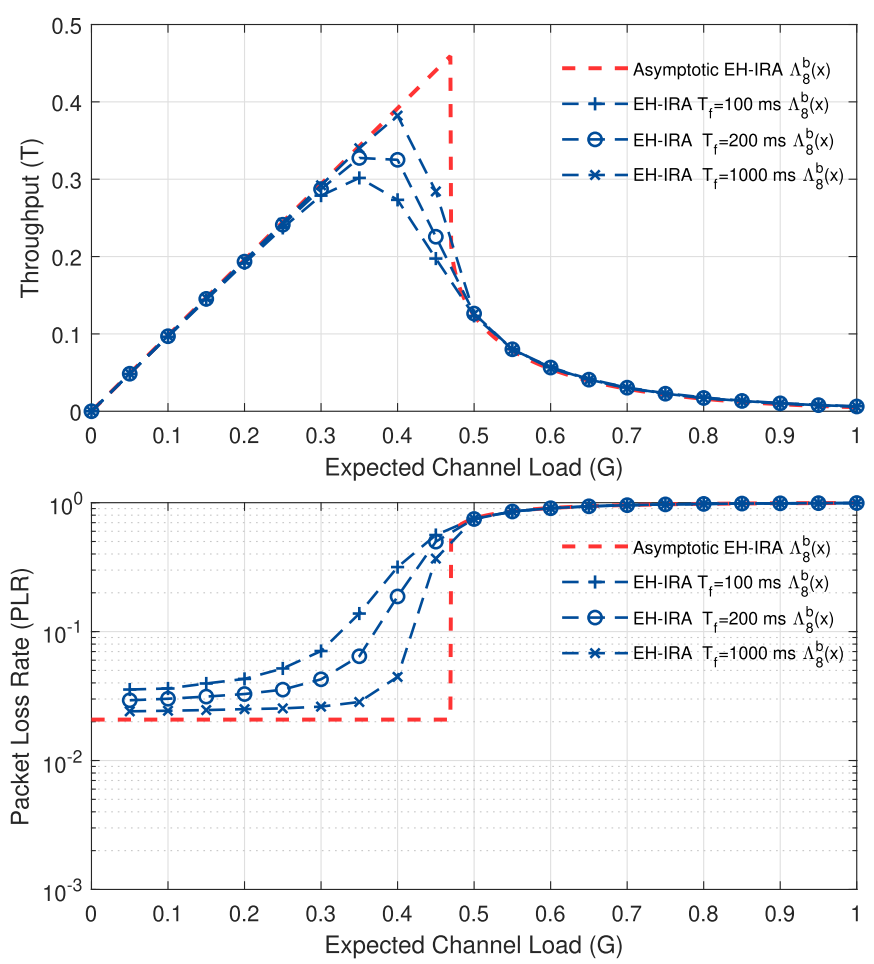

Fig. 9. Asymptotic analysis of EH-IRA with $B_{\max }=8, \alpha=10^{-1}$ and $\pi=10^{-2}$ for different virtual frame lengths $\left(T_{f}=100,200,1000 \mathrm{~ms}\right)$, $i_{\max }=20$.

decode all the transmitted packets through SIC. In addition, the finite-length performance of EH-IRARC also complies with the asymptotic analysis, and the proposed replica concatenation approach increases asymptotic throughput from 0.459 (EH-IRA) to 0.578 .

We examine the individual effects of the energy arrival rate $\alpha$ and activation probability $\pi$ on the system performance for both EH-IRA and EH-IRARC by using the repetition distribution $\Lambda_{8}^{b}(x)$. Fig. 10a depicts the maximum achievable asymptotic throughput, $T^{*}$, and the $P_{l}$ values for varying energy arrival rates for activity probability $\pi=10^{-2}$. Similarly, Fig. 10b illustrates the asymptotic performance for various activation probabilities for a fixed $\alpha$ value, $10^{-1}$. It can be seen from Fig. 10a that with increasing $\alpha$ values, $T^{*}$ increases with decreasing $P_{l}$, which is due to the fact the more energy is harvested from the environment, making energy absence less likely. On the other hand, as shown in Fig. 10b, as the activity probability $\pi$ increases, the system performance deteriorates because the user nodes become active more frequently, which results in shorter inactive periods for energy harvesting. It should also be noted that, the ratio $\alpha / \pi^{18}$ denotes the expected number of energy arrivals during an inactive state. If this ratio is too low (e.g., $\alpha / \pi<5$ ) the system performance deteriorates severely because the user nodes experience more energy shortages, and they can only transmit a limited number of replicas. For larger values of this ratio (e.g., $\alpha / \pi \geq 5$ ), the improvements are minor due to the limited battery capacity, as most of the energy arrivals are lost.

\footnotetext{
$181 / \pi$ denotes the expected number of frames until the user becomes active. Also, $\alpha$ denotes the expected number of energy arrivals for each frame. Hence, $\alpha / \pi$ represents total number of energy arrivals during an inactive state.
}

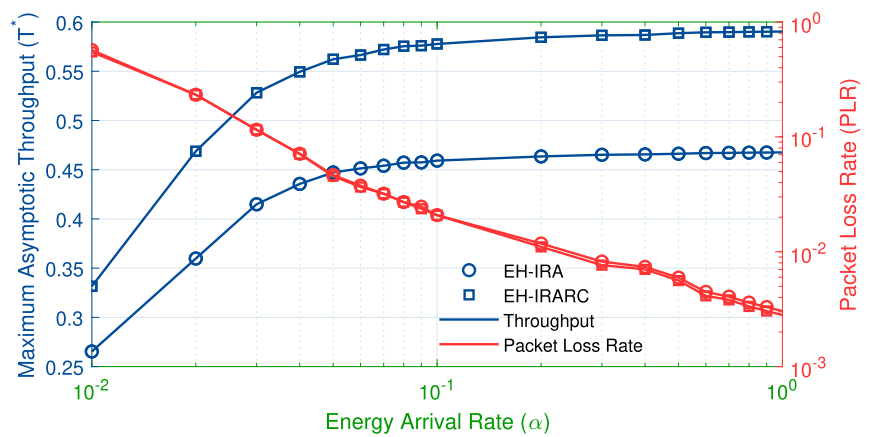

(a)

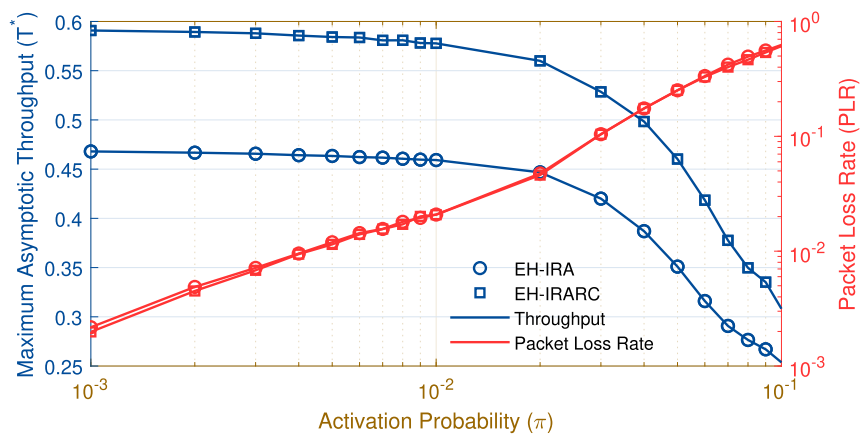

(b)

Fig. 10. Asymptotic performance of EH-IRA and EH-IRARC with $\Lambda_{8}^{b}(x)$ for different energy arrival rates and activation probabilities.

Lastly, EH-IRARC outperforms the EH-IRA in terms of the throughput by allowing higher channel loads, however, the $P_{l}$ curves are very close to each other since the packet losses are dominated by the user nodes with energy absence which is the same for both schemes.

We also show the advantages of the optimal transmission policy over two other alternatives: 1) Greedy transmission policy: User nodes consume all the energy in the battery by transmitting the maximum possible number of replicas, i.e., transmission probabilities for the greedy policy can be written as, $\gamma_{k}^{l}=1$ if $l=k$ and 0 otherwise for $k \in$ $\left\{0,1, \ldots, B_{\max }\right\}$,2) Uniform transmission policy: User nodes choose the number of replicas uniformly, i.e., $\gamma_{k}^{l}=\frac{1}{(k+1)}$ for $k \in\left\{0,1, \ldots, B_{\max }\right\}$. For example, if a battery contains 3 units of energy, in the greedy policy, a node transmits 3 replicas; whereas in the uniform policy, a node can send 0 , 1,2 or 3 replicas with probability, $1 / 4$ each. We provide the optimal transmission probabilities for $B_{\max }=8, \alpha=10^{-1}$ and $\pi=10^{-2}$ as, $\gamma_{0}^{0}=1, \gamma_{1}^{0}=1, \gamma_{2}^{2}=1, \gamma_{3}^{2}=0.992$, $\gamma_{3}^{3}=0.008, \gamma_{4}^{2}=1, \gamma_{5}^{2}=0.982, \gamma_{5}^{3}=0.018, \gamma_{6}^{2}=0.565$, $\gamma_{6}^{3}=0.435, \gamma_{7}^{2}=0.443, \gamma_{7}^{3}=0.026, \gamma_{7}^{4}=0.188, \gamma_{7}^{6}=$ $0.343, \gamma_{8}^{2}=0.436, \gamma_{8}^{3}=0.229, \gamma_{8}^{6}=0.302, \gamma_{8}^{8}=0.033$, and zero otherwise.

We present the resulting repetition distributions corresponding to the respective transmission policies in Table VI. The asymptotic performance of the transmission policies along with the finite length simulation results for EH-IRA is illustrated in Fig. 11. In the greedy policy, users consume all the energy in the battery which results in the distributions with higher probabilities in more replicas. This approach is 
TABLE VI

Optimized Distributions OF DifFEREnT TRANSMission Policies GIVEN $B_{\max }=8, \alpha=10^{-1}$ AND $\pi=10^{-2}$

\begin{tabular}{|c|c|c|}
\hline \hline Transmission Policy & User Degree Distributions $\Lambda(x)$ & EH-IRA $\left(T^{*}\right)$ \\
\hline \hline Greedy & $\Lambda_{g}(x)=0.1 x^{0}+0.08 x^{1}+0.07 x^{2}+0.07 x^{3}+0.06 x^{4}+0.06 x^{5}+0.05 x^{6}+0.05 x^{7}+0.46 x^{8}$ & 0.343 \\
\hline Uniform & $\Lambda_{u}(x)=0.16 x^{0}+0.14 x^{1}+0.13 x^{2}+0.12 x^{3}+0.11 x^{4}+0.1 x^{5}+0.09 x^{6}+0.08 x^{7}+0.07 x^{8}$ & 0.422 \\
\hline Optimal & $\Lambda_{o}(x)=0.02 x^{0}+0.53 x^{2}+0.19 x^{3}+0.01 x^{4}+0.2 x^{6}+0.03 x^{7}+0.02 x^{8}$ & 0.459 \\
\hline \hline
\end{tabular}

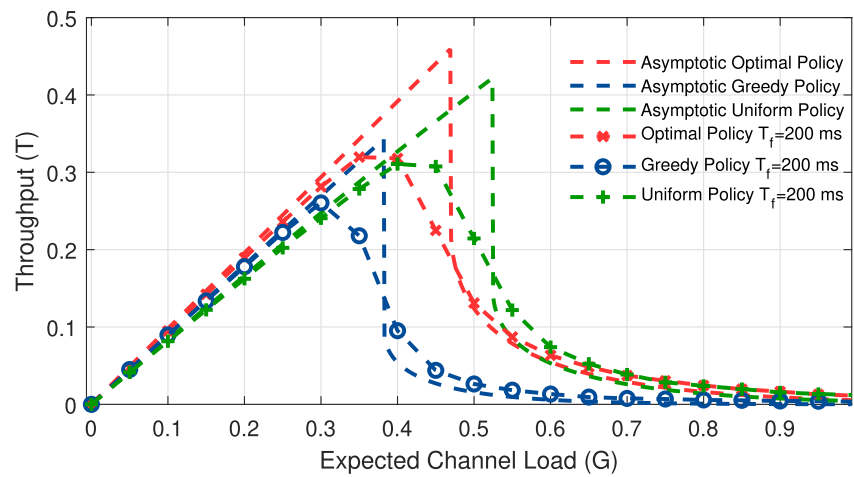

Fig. 11. Asymptotic throughput performance of greedy, uniform and optimal policies for EH-IRA with $B_{\max }=8, \alpha=10^{-1}$ and $\pi=10^{-2}$ with VF length $200 \mathrm{~ms}$ and $i_{\max }=20$.

not favorable for the system performance since collisions may become excessive. ${ }^{19}$ In the uniform policy, the corresponding repetition distribution provides better throughput than the greedy policy as it saves energy for the following rounds and less collisions occur thanks to the uniform selection. On the other hand, it leads to higher $P_{l}$ due to the users selecting to transmit no replicas. Further, the uniform policy can offer better throughput values for higher loads, since no replica transmission is more probable compared to the optimal one. However, this is not a desired behavior, since it is always possible to increase maximum load values $G^{*}$ with more packet loss keeping the throughput the same. The optimal policy minimizes the percentage of users suffering from the energy absence, which in fact results in a smaller $P_{l}$, and provides better throughput performance compared to the alternatives.

\section{CONCLUSION}

In this paper, we proposed an asynchronous RA scheme, namely IRA, as an extension to CRA by allowing varying repetition rates, in a similar way to IRSA as a generalization of CRDSA. The newly developed asymptotic analysis for both CRA and IRA shows that optimized irregular distributions perform remarkably better than the regular ones. We also proposed an enhancement to CRA/IRA by adding replica concatenation capability, which allows for merging the clean parts of (two) replicas. The analysis developed for the enhanced CRA/IRA scheme shows that a significant gain in the normalized throughput can be obtained. We also considered an energy limited scenario for users, and proposed

\footnotetext{
${ }^{19}$ We note that greedy policy suffers from transmitting maximum number of replicas (8) for modest and high load values. However, for lower load values, transmitting replicas with higher repetitions is beneficial by decreasing PLR.
}

a practical energy harvesting model for IRA and IRARC. We analyzed the proposed energy harvesting RA scheme considering stochasticity of the available energy, and derived an optimal transmission policy through the developed model aiming at improving the system performance. Finally, the asymptotic analysis results are corroborated with extensive finite length simulations. For future directions, we note that the developed analysis techniques provide a general framework, and they are amenable for further extensions; for instance, considering different channel models, asynchronous schemes with power imbalance among users, and coded asynchronous schemes could be possible.

\section{REFERENCES}

[1] T. Akyıldız, U. Demirhan, and T. M. Duman, "Asymptotic analysis of contention resolution ALOHA with replica concatenation," in Proc. IEEE Int. Conf. Commun. (ICC), Shanghai, China, May 2019, pp. 1-6.

[2] F. Boccardi, R. W. Heath, Jr., A. Lozano, T. L. Marzetta, and P. Popovski, "Five disruptive technology directions for 5G," IEEE Commun. Mag., vol. 52, no. 2, pp. 74-80, Feb. 2014.

[3] M. Agiwal, A. Roy, and N. Saxena, "Next generation 5G wireless networks: A comprehensive survey," IEEE Commun. Surveys Tuts., vol. 18, no. 3, pp. 1617-1655, 3rd Quart., 2016.

[4] E. Casini, R. De Gaudenzi, and O. R. Herrero, "Contention resolution diversity slotted ALOHA (CRDSA): An enhanced random access schemefor satellite access packet networks," IEEE Trans. Wireless Commun., vol. 6, no. 4, pp. 1408-1419, Apr. 2007.

[5] S. Sudevalayam and P. Kulkarni, "Energy harvesting sensor nodes: Survey and implications," IEEE Commun. Surveys Tuts., vol. 13, no. 3, pp. 443-461, 3rd Quart., 2011.

[6] N. Abramson, "The ALOHA system: Another alternative for computer communications," in Proc. Fall Joint Comput. Conf., 1970, pp. 281-285.

[7] L. G. Roberts, "ALOHA packet system with and without slots and capture," ACM SIGCOMM Comput. Commun. Rev., vol. 5, no. 2 , pp. 28-42, Apr. 1975.

[8] G. Choudhury and S. Rappaport, "Diversity ALOHA-A random access scheme for satellite communications," IEEE Trans. Commun., vol. 31, no. 3, pp. 450-457, Mar. 1983.

[9] G. Liva, "Graph-based analysis and optimization of contention resolution diversity slotted ALOHA," IEEE Trans. Commun., vol. 59, no. 2, pp. 477-487, Feb. 2011.

[10] E. Paolini, G. Liva, and M. Chiani, "Coded slotted ALOHA: A graphbased method for uncoordinated multiple access," IEEE Trans. Inf. Theory, vol. 61, no. 12, pp. 6815-6832, Dec. 2015.

[11] S. Gollakota and D. Katabi, "ZigZag decoding: Combating hidden terminals in wireless networks," in Proc. ACM SIGCOMM Conf. Data Commun., Seattle, WA, USA, 2008, pp. 159-170.

[12] J. Paek and M. J. Neely, "Mathematical analysis of throughput bounds in random access with ZIGZAG decoding," in Proc. 7th Int. Symp. Modeling Optim. Mobile, Ad Hoc, Wireless Netw. (WiOpt), Seoul, South Korea, Jun. 2009.

[13] S. Ogata and K. Ishibashi, "Application of ZigZag decoding in frameless ALOHA," IEEE Access, vol. 7, pp. 39528-39538, 2019.

[14] M. Oinaga, S. Ogata, and K. Ishibashi, "Design of coded ALOHA with ZigZag decoder,” IEEE Access, vol. 7, pp. 168527-168535, 2019.

[15] C. Stefanovic and P. Popovski, "ALOHA random access that operates as a rateless code," IEEE Trans. Commun., vol. 61, no. 11, pp. 4653-4662, Nov. 2013.

[16] K. R. Narayanan and H. D. Pfister, "Iterative collision resolution for slotted ALOHA: An optimal uncoordinated transmission policy," in Proc. 7th Int. Symp. Turbo Codes Iterative Inf. Process. (ISTC), Gothenburg, Sweden, Aug. 2012, pp. 136-139.

[17] F. Clazzer, E. Paolini, I. Mambelli, and C. Stefanovic, "Irregular repetition slotted ALOHA over the Rayleigh block fading channel with capture," in Proc. IEEE Int. Conf. Commun. (ICC), Paris, France, May 2017, pp. 1-6.

[18] A. Mengali, R. De Gaudenzi, and P.-D. Arapoglou, "Enhancing the physical layer of contention resolution diversity slotted ALOHA," IEEE Trans. Commun., vol. 65, no. 10, pp. 4295-4308, Oct. 2017.

[19] M. Ivanov, F. Brannstrom, A. G. I. Amat, and P. Popovski, "Error floor analysis of coded slotted ALOHA over packet erasure channels," IEEE Commun. Lett., vol. 19, no. 3, pp. 419-422, Mar. 2015. 
[20] Z. Sun, Y. Xie, J. Yuan, and T. Yang, "Coded slotted ALOHA for erasure channels: Design and throughput analysis," IEEE Trans. Commun., vol. 65 , no. 11, pp. 4817-4830, Nov. 2017.

[21] E. Sandgren, A. G. I. Amat, and F. Brännström, "On frame asynchronous coded slotted ALOHA: Asymptotic, finite length, and delay analysis," IEEE Trans. Commun., vol. 65, no. 2, pp. 691-704, Feb. 2017.

[22] A. G. I. Amat and G. Liva, "Finite-length analysis of irregular repetition slotted ALOHA in the waterfall region," IEEE Commun. Lett., vol. 22, no. 5, pp. 886-889, May 2018.

[23] C. Kissling, "Performance enhancements for asynchronous random access protocols over satellite," in Proc. IEEE Int. Conf. Commun. (ICC), Kyoto, Japan, Jun. 2011, pp. 1-6.

[24] F. Clazzer and C. Kissling, "Enhanced contention resolution ALOHAECRA," in Proc. 9th Int. ITG Conf. Syst. Commun. Coding (SCC), Munich, Germany, vol. 9, Nov. 2013, pp. 1-6.

[25] R. De Gaudenzi, O. del Rio Herrero, G. Acar, and E. G. Barrabes, "Asynchronous contention resolution diversity ALOHA: Making CRDSA truly asynchronous," IEEE Trans. Wireless Commun., vol. 13, no. 11, pp. 6193-6206, Nov. 2014.

[26] F. Clazzer, C. Kissling, and M. Marchese, "Enhancing contention resolution ALOHA using combining techniques," IEEE Trans. Commun., vol. 66, no. 6, pp. 2576-2587, Jun. 2018.

[27] T. Akyildiz and T. M. Duman, "Irregular repetition ALOHA with packet length diversity," in Proc. IEEE Global Commun. Conf. (GLOBECOM), Waikoloa, HI, USA, Dec. 2019, pp. 1-6.

[28] M. Moradian and F. Ashtiani, "Throughput analysis of a slotted alohabased network with energy harvesting nodes," in Proc. IEEE 23rd Int. Symp. Pers., Indoor Mobile Radio Commun. (PIMRC), Sydney, NSW, Australia, Sep. 2012, pp. 351-356.

[29] M. Moradian and F. Ashtiani, "Sum throughput maximization in a slotted aloha network with energy harvesting nodes," in Proc. IEEE Wireless Commun. Netw. Conf. (WCNC), Istanbul, Turkey, Apr. 2014 pp. $1585-1590$.

[30] J. Jeon and A. Ephremides, "On the stability of random multiple access with stochastic energy harvesting," IEEE J. Sel. Areas Commun., vol. 33, no. 3, pp. 571-584, Mar. 2015

[31] F. Iannello, O. Simeone, and U. Spagnolini, "Medium access control protocols for wireless sensor networks with energy harvesting," IEEE Trans. Commun., vol. 60, no. 5, pp. 1381-1389, May 2012.

[32] F. Vázquez-Gallego, J. Alonso-Zarate, and L. Alonso, "Reservation dynamic frame slotted-ALOHA for wireless M2M networks with energy harvesting," in Proc. IEEE Int. Conf. Commun. (ICC), London, U.K., Jun. 2015, pp. 5985-5991.

[33] F. Iannello, O. Simeone, P. Popovski, and U. Spagnolini, "Energy groupbased dynamic framed ALOHA for wireless networks with energy harvesting," in Proc. 46th Annu. Conf. Inf. Sci. Syst. (CISS), Princeton, NJ, USA, Mar. 2012, pp. 1-6.

[34] D. Shaviv, P.-M. Nguyen, and A. Özgür, "Capacity of the energyharvesting channel with a finite battery," IEEE Trans. Inf. Theory, vol. 62, no. 11, pp. 6436-6458, Nov. 2016.

[35] U. Demirhan and T. M. Duman, "Energy-harvesting irregular repetition slotted ALOHA with unit-sized battery," in Proc. IEEE Int. Conf. Commun. (ICC), Kansas City, MO, USA May 2018, pp. 1-6.

[36] U. Demirhan and T. M. Duman, "Irregular repetition slotted ALOHA with energy harvesting nodes," IEEE Trans. Wireless Commun., vol. 18, no. 9, pp. 4505-4517, Sep. 2019.

[37] M. Abramowitz and I. A. Stegun, Handbook of Mathematical Functions With Formulas, Graphs, and Mathematical Tables. New York, NY, USA: Dover, 1964.
[38] R. Storn and K. Price, "Differential evolution-A simple and efficient heuristic for global optimization over continuous spaces," J. Global Optim., vol. 11, no. 4, pp. 341-359, 1997.

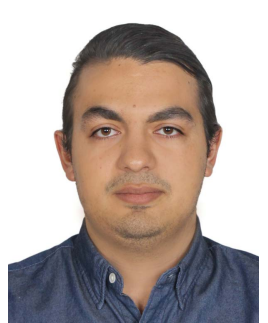

Talha Akyldız (Student Member, IEEE) received the B.S. and M.S. degrees in electrical and electronics engineering from Bilkent University, Ankara, Turkey, in 2017 and 2020, respectively. $\mathrm{He}$ is currently pursuing the Ph.D. degree with the Department of Electrical Engineering and Computer Science, University of Michigan, Ann Arbor, MI, USA. His research interests include the general area of wireless communications, with an emphasis in random access protocols and $5 \mathrm{G}$ and beyond.

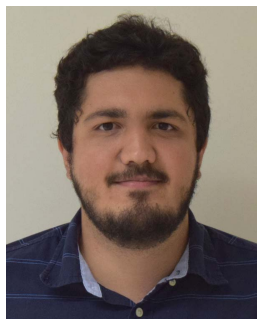

Umut Demirhan (Graduate Student Member, IEEE) received the B.S. and M.S. degrees in electrical and electronics engineering from Bilkent University, Ankara, Turkey, in 2015 and 2017, respectively. $\mathrm{He}$ is currently pursuing the Ph.D. degree in electrical engineering with Arizona State University, Tempe, AZ, USA. His current research interests include machine learning, optimization, and wireless communications with a particular focus on machine learning applications at the wireless edge.

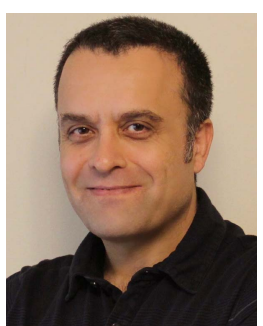

Tolga M. Duman (Fellow, IEEE) received the B.S. degree in electrical engineering from Bilkent University, Ankara, Turkey, in 1993, and the M.S. and $\mathrm{Ph} . \mathrm{D}$. degrees in electrical engineering from Northeastern University, Boston, MA, USA, in 1995 and 1998, respectively. He is currently a Professor with the Electrical and Electronics Engineering Department, Bilkent University. Prior to joining Bilkent University in September 2012, he was a Professor with the School of ECEE, Arizona State University. His current research interests include systems, with particular focus on communication and signal processing, including wireless and mobile communications, coding/modulation, coding for wireless communications, data storage systems, and underwater acoustic communications. He was a recipient of the National Science Foundation CAREER Award and the IEEE Third Millennium Medal. He is currently the Editor-in-Chief of the IEEE TRANSACTIONS ON COMMUNICATIONS. 